\title{
COMPUTATIONAL MODELING OF UNCERTAINTY IN TIME-DOMAIN ELECTROMAGNETICS*
}

\author{
C. CHAUVIÈRE ${ }^{\dagger}$, J. S. HESTHAVEN ${ }^{\ddagger}$, AND L. LURATI ${ }^{\ddagger}$
}

\begin{abstract}
We discuss computationally efficient ways of accounting for the impact of uncertainty, e.g., lack of detailed knowledge about sources, materials, shapes, etc., in computational time-domain electromagnetics. In contrast to classic statistical Monte Carlo-based methods, we explore a probabilistic approach based on high-order accurate expansions of general stochastic processes. We show this to be highly efficient and accurate on both one- and two-dimensional examples, enabling the computation of global sensitivities of measures of interest, e.g., radar-cross-sections (RCS) in scattering applications, for a variety of types of uncertainties.
\end{abstract}

Key words. Maxwell's equations, discontinuous Galerkin methods, uncertainty quantification, chaos expansion

AMS subject classifications. 65N35, 65C05, 65C20, 65C30

DOI. $10.1137 / 040621673$

1. Introduction. While computational methods have become increasingly refined and accurate, their reliance on exact data, e.g., complete descriptions of geometries, materials, sources, etc., is emerging as a bottleneck in the modeling of problems of realistic complexity. For instance, if one attempts to model an experiment, a classic computational approach would require knowledge of a degree of detail which is unrealistic and often impossible to obtain, e.g., one cannot hope to control all elements of an experiment, measure all details of an initial condition or geometry, know the microstructure of all materials, etc.

The usual approach to dealing with this lack of knowledge or uncertainty is to simply assume some mean parameters and compute the corresponding solution. If the solution is robust to parameter variation, this is indeed a reasonable approach. However, for general problems, where the sensitivity of parts of the solution can be significant, a solution based on mean parameters is not likely to match very well with experiments and, thus, is not a good predictive tool. An experimentalist would naturally deal with this exact problem by repeating the experiments and, subsequently, compute not only mean results but also error bars - reflecting, at least partly, the sensitivity of the solution.

It is natural to strive to achieve similar abilities in computational modeling effort, e.g., compute solutions or measures of interest and their associated sensitivities. Clearly, if a particular measured entity is highly sensitive there is no reason to expect that this matches experiments exactly. Thus, we would like to be able to model the impact of the uncertainty, assumed to have certain properties derived from experiments or otherwise, on the computed results, essentially resulting in an ensample of

* Received by the editors December 28, 2004; accepted for publication (in revised form) November 30, 2005; published electronically May 26, 2006.

http://www.siam.org/journals/sisc/28-2/62167.html

${ }^{\dagger}$ Laboratoire de Mathématiques, Université Blaise Pascal, 63177 Aubière Cedex, France (Cedric. Chauviere@math.univ-bpclermont.fr).

${ }^{\ddagger}$ Division of Applied Mathematics, Brown University, Providence, RI 02912 (Jan.Hesthaven@ brown.edu, laural@dam.brown.edu). The work of the second author was partly supported by NSF Career Award DMS0132967, AFOSR contract FA9550-04-1-0072, and the Alfred P. Sloan Foundation through a Sloan Research Fellowship. The work of the third author was supported by AFOSR contract FA9550-04-1-0072. 
possible solution values with an associated probability. This would immediately enable the computation of statistical moments, e.g., means and variances, and of other valuable information about the sensitivity of solutions and derived quantities.

In this paper we pursue this goal and present a systematic, accurate, and efficient way of addressing this type of problem, essentially enabling one to compute with an ensample of data and, subsequently, obtain a full space-time ensample of solutions with an associated probability density. It is important to realize that is this not a simple situation, since solutions may vary nonlinearly in the uncertainty due to stochastic correlations even if the deterministic problem is linear, e.g., Maxwell's equations.

A standard way of addressing problems of the type mentioned in the above is through Monte Carlo sampling [13], e.g., run a deterministic code a large number of times and, subsequently, extract the statistics of interest. The main problem with this approach is the very slow convergence rate, $\mathcal{O}\left(N^{-1 / 2}\right)$, with $N$ being the number of samples, which makes even the computation of mean solutions expensive and accurate recovery of higher moments, e.g., variances, prohibitive. As we shall see through examples, the techniques proposed here suggests that a very considerable reduction is possible without impacting the accuracy.

Our platform on which to demonstrate this approach is the time-domain Maxwell's equations, solved using a high-order accurate discontinuous Galerkin method [9]. However, the basic elements of the formulation are general and can be used with any computational kernel.

What remains of the paper is organized as follows. In the next two sections, we recall the deterministic Maxwell's equations in the time domain and we give some details of its space discretization using a high-order discontinuous Galerkin method. In section 4 we continue with an introduction to homogeneous chaos and stochastic collocation methods, enabling the transition from deterministic to stochastic modeling. In the same section, we explain how to model uncertainties and how to extract statistics of interest (i.e., mean and variance) from the computed stochastic solution. This sets the stage for numerous examples presented in section 5, comparing the two approaches and validating the general approach. In section 6 we conclude and offer some suggestions for continued research in this direction.

2. Maxwell's equations. Let us consider a general domain $\Omega$ and let $\mathbf{E}^{s}$ and $\mathbf{H}^{s}$ denote the scattered electric and magnetic fields, respectively. With $\varepsilon(\mathbf{x})$ and $\mu(\mathbf{x})$ being the local permittivity and permeability, and $\sigma(\mathbf{x})$ the conductivity of the media, the time-dependent Maxwell's equations in the scattered field formulation are given as

$$
\begin{aligned}
\epsilon \frac{\partial \mathbf{E}^{s}}{\partial t} & =\nabla \times \mathbf{H}^{s}+\sigma \mathbf{E}^{s}+\mathbf{S}^{E}, \\
\mu \frac{\partial \mathbf{H}^{s}}{\partial t} & =-\nabla \times \mathbf{E}^{s}+\mathbf{S}^{H},
\end{aligned}
$$

where, as is common in time-domain schemes, we have neglected divergence constraints, assuming that these amount to constraints on the initial conditions.

The source terms, $\mathbf{S}^{E}$ and $\mathbf{S}^{H}$, appearing on the right-hand side of (2.1)-(2.2), take the form

$$
\begin{aligned}
& \mathbf{S}^{E}=-\left(\epsilon-\epsilon^{i}\right) \frac{\partial \mathbf{E}^{i}}{\partial t}+\left(\sigma-\sigma^{i}\right) \mathbf{E}^{i} \\
& \mathbf{S}^{H}=-\left(\mu-\mu^{i}\right) \frac{\partial \mathbf{H}^{i}}{\partial t}
\end{aligned}
$$


where the incident field $\left(\mathbf{E}^{i}, \mathbf{H}^{i}\right)$ is a solution to Maxwell's equations in a media of permittivity, permeability, and conductivity $-\varepsilon^{i}(\mathbf{x}), \mu^{i}(\mathbf{x}), \sigma^{i}(\mathbf{x})$, respectively.

We now turn our attention to boundary conditions. Along a perfect electric conductor (PEC), the boundary conditions on the electric field are

$$
\hat{\mathbf{n}} \times \mathbf{E}^{t}=\mathbf{0},
$$

where $\hat{\mathbf{n}}$ is the outward pointing normal vector at the surface and $\mathbf{E}^{t}=\mathbf{E}^{i}+\mathbf{E}^{s}$ is the total field. For the total magnetic field $\mathbf{H}^{t}$, the condition is

$$
\mathbf{H}^{t} \cdot \hat{\mathbf{n}}=0 .
$$

Along the interface of two dielectric bodies, denoted by the subscripts 1 and 2, we have

$$
\hat{\mathbf{n}} \times\left(\mathbf{E}_{1}^{s}-\mathbf{E}_{2}^{s}\right)=\mathbf{0} \text { and } \hat{\mathbf{n}} \times\left(\mathbf{H}_{1}^{s}-\mathbf{H}_{2}^{s}\right)=\mathbf{0} ;
$$

i.e., all tangential components are continuous.

3. Numerical scheme for the deterministic case. Before turning our attention to the case including uncertainties, let us briefly describe the computational methods used for solving Maxwell's equations in the physical space. We use a discontinuous Galerkin formulation; the solution will be discontinuous between elements. This offers a number of advantages over widely used alternative techniques, e.g., geometric flexibility through fully unstructured grids, high-order accuracy to enable accurate wave propagation over long distance with a coarse resolution, and very high computational efficiency through explicit time stepping and high parallel performance. The method has been discussed, analyzed, and validated extensively $[9,10,11,12]$, and we shall simply sketch its main components.

We rewrite Maxwell's equations (2.1)-(2.2) in conservation form

$$
Q \frac{\partial \mathbf{q}}{\partial t}+\nabla \cdot \mathbf{F}(\mathbf{q})=\mathbf{S}
$$

where $\mathbf{q}$ is the state vector formed by the scattered electric field and the magnetic field

$$
\mathbf{q}=\left(\begin{array}{c}
\mathbf{E} \\
\mathbf{H}
\end{array}\right)
$$

and the components of the tensor $\mathbf{F}$ are defined by

$$
\mathbf{F}_{i}(\mathbf{q})=\left(\begin{array}{c}
-\mathbf{e}_{i} \times \mathbf{H} \\
\mathbf{e}_{i} \times \mathbf{E}
\end{array}\right),
$$

where $\mathbf{e}_{i}$ denotes the Cartesian unit vectors. On the right-hand side of (3.1), $\mathbf{S}=$ $\left[\mathbf{S}^{E}, \mathbf{S}^{H}\right]$ is the source term, which depends on the incident field, and the material matrix $Q$ is a diagonal matrix with values $(\epsilon, \epsilon, \epsilon, \mu, \mu, \mu)$ on its diagonal.

We assume that the computational domain, $\Omega$, is tessellated by triangles or tetrahedrons, $D$, and we represent the local solution $\mathbf{q}_{N}$ as

$$
\mathbf{q}_{N}(\mathbf{x}, t)=\sum_{i=1}^{N} \widetilde{\mathbf{q}}_{i}(t) L_{i}(\mathbf{x}),
$$


where $\widetilde{\mathbf{q}}_{i}$ reflects nodal values, defined on the element, and $L_{i}(\mathbf{x})$ signifies an $n$th order Lagrange polynomial, associated with grid points; see $[9,10,11,12]$ for details.

The discrete solution, $\mathbf{q}_{N}$, of Maxwell's equations is required to satisfy

$$
\int_{D}\left(Q \frac{\partial \mathbf{q}_{N}}{\partial t}+\nabla \cdot \mathbf{F}\left(\mathbf{q}_{N}\right)-\mathbf{S}_{N}\right) L_{i}(\mathbf{x}) d \mathbf{x}=\oint_{\partial D} \hat{\mathbf{n}} \cdot\left[\mathbf{F}\left(\mathbf{q}_{N}\right)-\mathbf{F}^{*}\right] L_{i}(\mathbf{x}) d \mathbf{x} .
$$

In (3.5), $\mathbf{F}^{*}$ denotes a numerical flux and $\hat{\mathbf{n}}$ is an outward pointing unit vector defined at the boundary $\partial D$ of the element $D$. Note that this is an entirely local formulation, and relaxing the continuity of the elements decouples the elements, resulting in a block-diagonal global mass matrix which can be trivially inverted in preprocessing. The price to pay for this is the additional degrees of freedom needed to support the local basis functions. For high-order elements, this is, however, only a small fraction of the total number of degrees of freedom. Given the linearity of Maxwell's equations, and for stability reasons, it is natural to use an upwinding flux which takes the form [9]

$$
\hat{\mathbf{n}} \cdot\left[\mathbf{F}\left(\mathbf{q}_{N}\right)-\mathbf{F}^{*}\right]=\left(\begin{array}{c}
\bar{Z}^{-1} \hat{\mathbf{n}} \times\left(\hat{\mathbf{n}} \times\left[\mathbf{E}_{N}\right]-Z^{+}\left[\mathbf{H}_{N}\right]\right) \\
\bar{Y}^{-1} \hat{\mathbf{n}} \times\left(\hat{\mathbf{n}} \times\left[\mathbf{E}_{N}\right]-Z^{+}\left[\mathbf{H}_{N}\right]\right)
\end{array}\right),
$$

where the bracket $[\mathbf{q}]=\mathbf{q}^{-}-\mathbf{q}^{+}$denotes the jump in the field values $\left(\mathbf{q}^{-}\right.$is the local value and $\mathbf{q}^{+}$is the neighboring value) across an interface, $Z^{ \pm}$is the local impedance, and $Y^{ \pm}$is the local conductance,

$$
Z^{ \pm}=\frac{1}{Y^{ \pm}}=\sqrt{\frac{\mu^{ \pm}}{\epsilon^{ \pm}}}
$$

$\bar{Z}$ and $\bar{Y}$ in (3.6) are the sums

$$
\bar{Z}=Z^{+}+Z^{-}, \quad \bar{Y}=Y^{+}+Y^{-} .
$$

After discretization of the operators and evaluation of the integrals appearing in (3.5), the problem can be rewritten in matrix-vector form (see [10] for details)

$$
Q M \frac{d \mathbf{q}_{N}}{d t}+S \cdot \mathbf{F}_{N}-M \mathbf{S}_{N}=F \hat{\mathbf{n}} \cdot\left[\mathbf{F}_{N}-\mathbf{F}^{*}\right] .
$$

The matrices $M, S$, and $F$ represent the local mass-, stiffness-, and face-integration matrices, respectively, the exact entries of which can be found in [9], where it is also discussed how to compute these local operators efficiently and accurately. Note that the local nature of the scheme allows for the use of an explicit solver for the time discretization of (3.9). This is traditionally done using an explicit Runge-Kutta method, although suitable alternatives are plentiful.

4. Accounting for the uncertainty. To deal with the actual lack of detailed knowledge leading to the uncertainty, we must make some educated guesses - a modelabout the nature of the possible variations in the data. These models can be based on pure speculation, on measured data, or on other available information. Once this is done, however, we must introduce this into the computational approach in an efficient, accurate, and robust manner.

Consider, as a simple example, the wave equation

$$
\frac{\partial u}{\partial t}+a(\theta) \frac{\partial u}{\partial x}=0,
$$


where $\theta$ is a random parameter with some associated probability density function (PDF). Thus, this represents an ensemble of wave problems, each with an individual phase speed occurring with a probability given by the PDF; i.e., the solution $u$ is not only a function of $(x, t)$ but also of $\theta$, and the equation is a stochastic wave equation.

The actual form of $a(\theta)$ may not be known, causing the introduction of the uncertainty. However, it seems quite reasonable that in many situations one can measure or conjecture the average of $a$ and perhaps its variation; i.e., one can reasonably assume that $a(\theta)$ varies in a certain way related to a normal distribution with a given mean and variance.

The question remains, however, how this uncertainty will affect the solution, $u(x, t, \theta)$, and, more often, its moments such as the mean and the variance. This is not a trivial question, as the uncertainty essentially renders the simple linear problem considerably more complex due to possible stochastic dependence between $a$ and $u$.

One could naturally solve the above problem for a large number of values of $\theta$, taken from a proper distribution, and, subsequently, compute the appropriate moments. This is the essence of a Monte Carlo method and suffers from a very slowly converging result as $1 / \sqrt{N}$, with $N$ being the number of samples. If higher-order moments, e.g., the variance or sensitivity of the result, are required, this is often prohibitive.

In the above example, the uncertainty enters through the phase speed. However, as we shall see shortly, dealing with uncertainty in initial conditions, boundary conditions, sources, or computational domain/geometries can be done in an entirely similar fashion.

In this section, we shall discuss two variations, referred to as stochastic Galerkin and collocation, respectively, of the same underlying result. This enables one to discretize stochastic partial differential equations (SPDEs) to recover systems of deterministic problems which we can subsequently solve with the methods discussed in section 3. As we shall experience through examples, both techniques offer excellent accuracy at a severely reduced computational cost as compared to straightforward Monte Carlo methods.

4.1. The homogeneous chaos expansion. The key result on which we shall rely is due to Wiener [16] (see also Cameron and Martin [3], Schoutens [14], and Ghanem and Spanos [7]) and shows that the chaos expansion can be used to approximate any functional in $L^{2}(\Omega, \mathcal{P})$, where $\mathcal{P}$ is a Gaussian measure on $\Omega$. For such a random variable $X(\theta)$, the chaos expansion is written as

$$
\begin{aligned}
X(\theta)= & a_{0} H_{0} \\
& +\sum_{i_{1}=1}^{d} a_{i_{1}} H_{1}\left(\xi_{i_{1}}(\theta)\right) \\
& +\sum_{i_{1}=1}^{d} \sum_{i_{2}=1}^{i_{1}} a_{i_{1} i_{2}} H_{2}\left(\xi_{i_{1}}(\theta), \xi_{i_{2}}(\theta)\right) \\
& +\sum_{i_{1}=1}^{d} \sum_{i_{2}=1}^{i_{1}} \sum_{i_{3}=1}^{i_{2}} a_{i_{1} i_{2} i_{3}} H_{3}\left(\xi_{i_{1}}(\theta), \xi_{i_{2}}(\theta), \xi_{i_{3}}(\theta)\right) \\
& +\cdots
\end{aligned}
$$

where $\xi=\left(\xi_{1}(\theta), \ldots, \xi_{d}(\theta)\right)$ represents $d$ independent Gaussian variables with zero mean and unit variance, each depending on the random event $\theta$, and $H_{n}$ are the 
multivariate Hermite polynomials defined as

$$
H_{n}\left(\xi_{i_{1}}(\theta), \ldots, \xi_{i_{n}}(\theta)\right)=e^{\frac{1}{2} \xi^{T} \xi}(-1)^{n} \frac{\partial^{n}}{\partial \xi_{i_{1}}, \ldots, \partial \xi_{i_{n}}} e^{-\frac{1}{2} \xi^{T} \xi} .
$$

The number of terms in the expansion (4.2) grows as

$$
P=\frac{(n+d) !}{n ! d !},
$$

where $n$ is the length of the Hermite expansion and $d$ is the dimension of the Gaussian random space. The polynomial homogeneous chaos expansion forms a complete orthogonal basis in the space of Gaussian variables, i.e.,

$$
\begin{aligned}
& \left\langle H_{i}\left(\xi_{i_{1}}(\theta), \ldots, \xi_{i_{n}}(\theta)\right), H_{j}\left(\xi_{j_{1}}(\theta), \ldots, \xi_{j_{n}}(\theta)\right)\right\rangle \\
& =\int_{R^{d}} \frac{H_{i}\left(\xi_{i_{1}}(\theta), \ldots, \xi_{i_{n}}(\theta)\right) H_{j}\left(\xi_{j_{1}}(\theta), \ldots, \xi_{j_{n}}(\theta)\right)}{\sqrt{(2 \pi)^{d}}} e^{-\frac{1}{2} \xi^{T} \xi} d \xi=i ! \delta_{i j},
\end{aligned}
$$

where $i$ denotes the multi-index $\left(i_{1}, \ldots, i_{n}\right)$ and $i !=i_{1} !, \ldots, i_{n} !$. Thus there is an intimate relation between the Hermite polynomials, orthogonal under the Gaussian weight, and the representation of random variables taken from a Gaussian distribution. One way of interpreting the homogeneous chaos expansion is that a general random variable can be expressed in terms of simpler Gaussian variables for which we can construct an efficient computational approach. Clearly, if the random variable is far from Gaussian, many terms in the expansion will be needed; i.e., $n$ must be large. An alternative is to use an expansion in terms of other random variables with an associated distribution closer to what is expected at input or output (see [14] for generalizations). For notational convenience, we can rewrite (4.2) in the form

$$
X(\theta)=\sum_{j=1}^{P} b_{j} \Psi_{j}(\xi),
$$

where there is a one-to-one correspondence between the functions $H_{i}$ and $\Psi_{j}$ and also between the coefficients $a_{i_{1}, \ldots, i_{p}}$ and $b_{j}$. In the case of a general stochastic process, these coefficients will be time dependent.

To model the impact of uncertainty on the propagation of electromagnetic waves, we include the randomness in the usual spatial-temporal dimensions; i.e., the electric field and the magnetic field become $\mathbf{E}(\mathbf{x}, t, \theta)$ and $\mathbf{H}(\mathbf{x}, t, \theta)$, reflecting that the fields are functions of $d$ independent random variables, $\left(\xi_{i_{1}}(\theta), \ldots, \xi_{i_{d}}(\theta)\right)$.

In the following we shall discuss in some detail how this can be utilized to construct an efficient computational method. For simplicity of the discussion, we assume in what follows that one Gaussian variable suffices to represent the process (i.e., $d=1$ ). However, the formulation is general and applies to problems requiring many random variables to describe the stochastic processes.

4.2. Stochastic Galerkin formulation. Using the chaos expansion, we can express $\mathbf{q}(\mathbf{x}, t, \theta)=(\mathbf{E}(\mathbf{x}, t, \theta), \mathbf{H}(\mathbf{x}, t, \theta))^{T}$ as

$$
\mathbf{q}(\mathbf{x}, t, \theta)=\sum_{i=1}^{P} \mathbf{q}^{i}(\mathbf{x}, t) \Psi_{i}(\theta) .
$$


We can write the computational scheme, which takes into account randomness in a general setting, as

$$
\left\{\begin{array}{l}
Q(\theta) M \frac{d \mathbf{q}_{N}}{d t}+S \cdot \mathbf{F}_{N}-M \mathbf{S}(\theta)_{N}=F \hat{\mathbf{n}} \cdot\left[\mathbf{F}_{N}-\mathbf{F}^{*}\right] \\
\mathbf{q}_{N}(\mathbf{x}, t=0, \theta)=\mathbf{f}(\mathbf{x}, \theta)
\end{array}\right.
$$

where the initial conditions are given by the function $\mathbf{f}=\mathbf{f}(\mathbf{x}, \theta)$ and the unknown vector $\mathbf{q}_{N}$ is given by (4.7). As a first step, we discretize (4.8) in the random space using a Galerkin approach. Multiplying (4.8) by a test function $\Psi_{k}(\theta)$, replacing $\mathbf{q}_{N}$ by its chaos expansion, and using the scalar product defined by (4.5), we obtain

$$
\forall k \in[1, P]: \sum_{i=1}^{P}\left\langle Q \Psi_{i}, \Psi_{k}\right\rangle M \frac{d \mathbf{q}_{N}^{i}}{d t}+k ! S \cdot \mathbf{F}_{N}^{k}-M \mathbf{S}_{N}^{k}=F \sum_{i=1}^{P} \hat{\mathbf{n}} \cdot\left[\mathbf{F}_{N}^{i}-\mathbf{F}^{i *}\right],
$$

where we have used property (4.5). The detailed expression of the terms on the right-hand side is

$$
\hat{\mathbf{n}} \cdot\left[\mathbf{F}_{N}^{i}-\mathbf{F}^{i *}\right]=\left(\begin{array}{c}
\left\langle\bar{Z}^{-1} \Psi_{i}, \Psi_{k}\right\rangle \hat{\mathbf{n}} \times \hat{\mathbf{n}} \times\left[\mathbf{E}^{i}\right]-\left\langle Z^{+} \bar{Z}^{-1} \Psi_{i}, \Psi_{k}\right\rangle \hat{\mathbf{n}} \times\left[\mathbf{H}^{i}\right] \\
\left\langle\bar{Y}^{-1} \Psi_{i}, \Psi_{k}\right\rangle \hat{\mathbf{n}} \times \hat{\mathbf{n}} \times\left[\mathbf{H}^{i}\right]+\left\langle Y^{+} \bar{Y}^{-1} \Psi_{i}, \Psi_{k}\right\rangle \hat{\mathbf{n}} \times\left[\mathbf{E}^{i}\right]
\end{array}\right) .
$$

Recall that $Z$ and $Y$ may depend on the material properties and, thus, may include uncertainty/randomness.

The initial conditions in (4.8) also need to be projected onto the chaos basis to give an initial condition for each mode of $\mathbf{q}_{N}^{i}$ in the chaos expansion, i.e.,

$$
\forall i \in[1, P]: \mathbf{q}_{N}^{i}(\mathbf{x}, t=0)=\frac{1}{i !}\left\langle\mathbf{f}(\mathbf{x}, \theta), \Psi_{i}\right\rangle .
$$

Once the vectors $\left\{\mathbf{q}_{N}^{i}\right\}_{1 \leq i \leq P}$ of the system (4.9) have been computed, we have available at every point in space an approximation to the probability density of the solution to the system.

Considering (4.9), we observe that we have managed to recast the general stochastic problem into a system of $P$ coupled deterministic problems which we can now discretize in space/time exactly as discussed in section 3-or in any other preferred way.

4.3. Stochastic collocation formulation. The idea of the stochastic collocation formulation is to replace the expression of the electric and magnetic fields (4.7) in the polynomial chaos expansion with a Lagrangian polynomial basis; i.e., we would have

$$
\mathbf{q}(\mathbf{x}, t, \theta)=\sum_{i=1}^{P} \mathbf{q}^{i}(\mathbf{x}, t) L_{i}(\theta),
$$

where $\left\{L_{i}(\theta)\right\}_{1 \leq i \leq P}$ forms a Lagrangian polynomial basis of degree $(P-1)$. In the deterministic community, (4.7) would be referred to as a modal expansion and (4.12) as a nodal expansion. Since $\theta$ in (4.12) is a Gaussian variable with zero mean and 


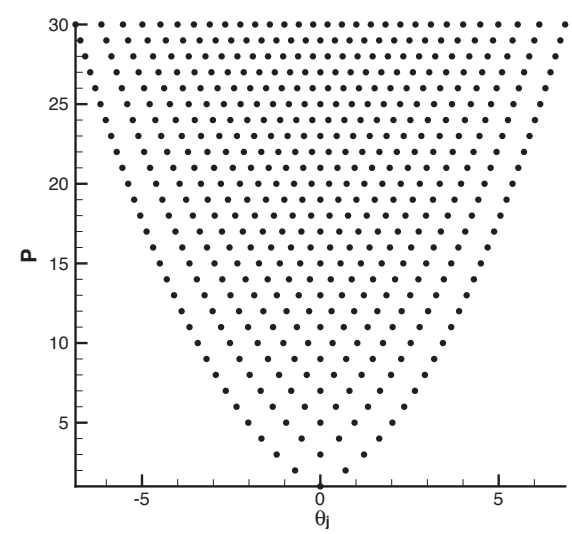

FIG. 4.1. Distribution of Gauss-Hermite collocation points for $P=1, \ldots, 30$.

unit variance, it seems natural to use Gauss-Hermite collocation points as the basis for the Lagrange polynomials, as that also enables accurate evaluation of integrals. Figure 4.1 shows the collocation $\left\{\theta_{j}\right\}_{1 \leq j \leq P}$ points for $P=1, \ldots, 30$. It should be noted that those points are more concentrated near zero, where the probability of the normal law is highest.

For reasons to be explained shortly, the Lagrange polynomials of (4.12) will not be based on $\theta_{j}$ but on $\sqrt{2} \theta_{j}$. Therefore, by the property of Lagrange polynomials $L_{i}\left(\sqrt{2} \theta_{j}\right)=\delta_{i j},(4.12)$ gives

$$
\mathbf{q}\left(\mathbf{x}, t, \sqrt{2} \theta_{j}\right)=\mathbf{q}^{j}(\mathbf{x}, t) .
$$

In the collocation formulation, we require that the residual of (4.8) be zero at collocation points $\left\{\sqrt{2} \theta_{j}\right\}_{1 \leq j \leq P}$, i.e.,

$$
\forall j \in[1, P]:\left\{\begin{array}{l}
Q^{j} M \frac{d \mathbf{q}_{N}^{j}}{d t}+S \cdot \mathbf{F}_{N}^{j}-M \mathbf{S}_{N}^{j}=F \hat{\mathbf{n}} \cdot\left[\mathbf{F}_{N}^{j}-\mathbf{F}^{j *}\right], \\
\mathbf{q}_{N}^{j}(\mathbf{x}, t=0)=\mathbf{f}\left(\mathbf{x}, \sqrt{2} \theta_{j}\right) .
\end{array}\right.
$$

The stochastic collocation method is essentially a deterministic or lattice Monte Carlo method with samples $\left\{\sqrt{2} \theta_{j}\right\}_{1 \leq j \leq P}$. The crucial difference is that a typical Monte Carlo simulation consisting of $P$ realizations will converge at the slow rate $1 / \sqrt{P}$, whereas the convergence of the stochastic collocation method is much faster, as will be shown by numerical examples (the interested reader can also refer to [17] for classical results in approximation theory). A simple analogy is that the approximation of a smooth function, the PDF, is done most efficiently by representing it by smooth polynomials based on points well suited for interpolation. In a simple Monte Carlo approach, the interpolation points are random, leading to a poor convergence rate.

We presented the stochastic collocation method for a random space of size $d=1$. The generalization to higher random spaces is straightforward, using tensor products of quadrature points. Note that for higher random spaces and for purposes of efficiency, it might be necessary to use sparse grid methods (see [17] for details).

4.4. A brief comparison and discussion of the two techniques. It is worth highlighting a few essential differences between the two ways of introducing the uncertainty into the model. Although both rely on the use of the chaos expansion, the 
two formulations lead to computationally different methods. In the Galerkin method, one recovers a system of $P$ coupled equations, even if a scalar equation is considered initially. While the coupling coefficients can be precomputed, thus reducing the cost, essential questions, such as well-posedness of the system, do not follow trivially from the well-posedness of the scalar equation. However, analysis confirming the well-posedness of the system can be found in [5].

For the collocation approach, however, these problems never arise as one solves $P$ decoupled problems, all of a type similar to that of the original problem. Thus, in terms of computational efficiency, the stochastic collocation method compares favorably to the stochastic Galerkin formulation.

On the other hand, there are situations in which the Galerkin formulation is superior to the collocation approach in terms of accuracy. To appreciate this, consider again (4.1) and assume, for simplicity, that the spatial direction is periodic. We use a Fourier spectral method in this direction; i.e., we assume

$$
u(x, t, \theta)=\sum_{|n| \leq N} \hat{u}_{n}(\theta, t) \exp (\text { inx }) .
$$

This yields the semidiscrete scheme

$$
\forall|n| \leq N: \frac{d \hat{u}_{n}(\theta, t)}{d t}+\tilde{a}(\theta) \hat{u}_{n}=0,
$$

with $\tilde{a}(\theta)=i n a(\theta)$.

If we now represent the orthogonal projection of $u$ onto the space spanned by the Hermite polynomial by $\mathcal{P}_{P} u$, we recover the error equation

$$
\frac{d}{d t} \varepsilon+\tilde{a}(\theta) \hat{u}_{n}-\mathcal{P}_{P}\left(\tilde{a}(\theta) \hat{u}_{n}\right)=0,
$$

where $\varepsilon(t)=\hat{u}_{n}-\mathcal{P}_{P} \hat{u}_{n}$ with

$$
\mathcal{P}_{P} \hat{u}_{n}=\sum_{i=1}^{P} \hat{u}_{n i}(t) H_{i}(\theta) .
$$

From classic approximation results for orthogonal polynomials [6], we recall

$$
\left\|u-\mathcal{P}_{P} u\right\|_{w} \leq P^{-k}\left\|u^{(k)}\right\|_{w}, \quad P>k \geq 0 .
$$

Here $\|\cdot\|_{w}$ is the Gaussian weighted inner product and $u^{(k)}$ reflects the $k$ th derivative of $u$, i.e., it is a simple measure of the smoothness of the probability density associated with $u$.

Integration in $\theta$ and in time immediately yields the error bound

$$
\|\varepsilon(t)\|_{w} \leq\|\varepsilon(0)\|_{w}+t P^{-k} \max _{s \in[0, t]}\left\|\left(\tilde{a} \hat{u}_{n}\right)^{(k)}(s)\right\|_{w}
$$

or

$$
\|\varepsilon(t)\|_{w} \leq P^{-k}\left(\left\|\hat{u}_{n}^{(k)}(0)\right\|_{w}+t \max _{s \in[0, t]}\left\|\left(\tilde{a} \hat{u}_{n}\right)^{(k)}(s)\right\|_{w}\right) .
$$

Thus, if $\hat{u}_{n}^{(k)}(0)$ and $\left(a \hat{u}_{n}\right)^{(k)}$ are well behaved, i.e., reasonably smooth, the scheme converges, although the details naturally depend on $\tilde{a}$ and the initial conditions. 
If we now consider the collocation formulation in exactly the same way, we shall need the interpolation estimate $[6]$

$$
\left\|u-\mathcal{I}_{P} u\right\|_{w} \leq P^{-k+1}\left\|u^{(k)}\right\|_{w}, \quad P>k \geq 1,
$$

where

$$
\mathcal{I}_{P} \hat{u}_{n}=\sum_{i=1}^{P} \hat{u}_{n}\left(\theta_{i}, t\right) L_{i}(\theta)
$$

is the Lagrange form of the chaos expansion as discussed above. This immediately yields the estimate

$$
\|\varepsilon(t)\|_{w} \leq P^{-k+1}\left(\left\|\hat{u}_{n}^{(k)}(0)\right\|_{w}+t \max _{s \in[0, t]}\left\|\left(\tilde{a} \hat{u}_{n}\right)^{(k)}(s)\right\|_{w}\right),
$$

provided $k \geq 1$.

Comparing the bounds in (4.15) and (4.16) highlights several properties of the two different formulations. We notice, as mentioned above, that the Galerkin formulation in general is more accurate than the collocation approach, in particular for problems of low regularity (small $k$ ); i.e., for problems with very low regularity $(k \leq 1)$ the collocation approach may well fail. Thus, for problems in which the probability density of $\hat{u}_{n}$ develops kinks or cusps, a Galerkin approach is likely to be advantageous. However, for problems in which both $a(\theta)$ and the probability density of the solution, $u$, are smooth in $\theta$, the two methods are essentially equivalent in terms of accuracy. As we shall see shortly, for the different types of applications considered here, the two methods behave in very similar ways in terms of accuracy.

The analysis also shows that the error grows linearly in time - at least for problems of simple wave type (4.1). We can obtain a further appreciation of the impact of this by considering the exact solution

$$
\hat{u}_{n}(\theta, t)=\hat{u}_{n}(\theta, 0) \exp (\text { ina }(\theta) t) .
$$

The length, $P$, of the chaos expansion must naturally be chosen to represent this solution adequately at all times. While this is easily accomplished for the initial conditions, $\hat{u}_{n}(\theta, 0)$, the other term reflects a wave, where the wavenumber, $a(\theta) n t$, grows with time. Again borrowing from classic results in approximation theory [8], we know that if we consider

$$
\exp (i k x)=\sum_{l=0}^{L} \hat{u}_{l} H_{l}(x),
$$

then exponential accuracy requires that

$$
\frac{2^{l}(l / 2) !}{k^{l}} \exp \left(\frac{l^{2}}{4}\right)>\exp \left(\frac{1}{2}\left(\frac{l \pi}{k p}\right)^{2}\right)
$$

at a fixed value of $x$. Here

$$
p=\frac{\lambda}{2 x / l}
$$


reflects the number of degrees of freedom used to represent the wave of wavelength $\lambda$.

Thus, if we want to accurately resolve waves within $x \in[-\pi, \pi]$, we find that $l p^{-1}=1$, and the requirement above is fulfilled for $l=p>6$. However, if we are interested in resolving the problem in $x \in[-2 \pi, 2 \pi]$, then $l p^{-1}=2$, and we obtain $l=2 p>15$, while $x \in[-4 \pi, 4 \pi]$ implies $l p^{-1}=4$ and the bound $l=4 p>44$.

These simple estimates have a few consequences for the methods discussed here. In particular, we find in the above that the effective wavenumber will grow linearly with time. This means that if we want to ensure an accurate representation of the local (in space) probability density, we shall generally expect that $P$, the number of terms in the chaos expansion, must grow linearly.

This requires, however, that the local PDF does not change too much over time; i.e., if it expands significantly in $\theta$ space beyond the initial range, we must ensure that it is well represented within a larger range of $\theta$ values, which requires, as seen above, a faster than linear growth. These arguments are independent of whether we consider the Galerkin or the collocation approach.

4.5. Computation of statistics. In practice, an expression of the stochastic fields in the form (4.7) or (4.12), containing a full space-time approximation to the local PDF, is of little use and often contains too much information. Quantities of interest - observables - are often macroscopic quantities, i.e., averages or variances of the fields. The way to extract those quantities depends on whether we have used a collocation formulation or a Galerkin formulation, and the procedure will also be different depending on whether we are interested in the statistics of linear or nonlinear functions of the electric or magnetic field. We shall sketch the different procedures in the next subsections, as this is an important component of the complete algorithm.

4.5.1. Statistics of linear quantities. As the treatment of the two methods is slightly different, we shall discuss each separately.

Stochastic Galerkin method. We assume that the solution $\mathbf{q}(\mathbf{x}, t, \theta)$ is available in the form (4.7), and we wish to extract some statistical information such as the average solution or its variance.

Let us first assume that we seek the moments of the solutions or a linear combination of them. Taking the average of (4.7), we get

$$
\begin{aligned}
\langle\mathbf{q}(\mathbf{x}, t, \theta), 1\rangle & =\sum_{i=1}^{P} \mathbf{q}^{i}(\mathbf{x}, t)\left\langle 1, \Psi_{i}(\theta)\right\rangle=\sum_{i=1}^{P} \mathbf{q}^{i}(\mathbf{x}, t)\left\langle\Psi_{1}(\theta), \Psi_{i}(\theta)\right\rangle \\
& =\sum_{i=1}^{P} \mathbf{q}^{i}(\mathbf{x}, t) \delta_{1 i}=\mathbf{q}^{1}(\mathbf{x}, t) .
\end{aligned}
$$

Thus, the average is simply the first mode in the chaos expansion. In a similar way, we can obtain the variance by first computing

$$
\begin{aligned}
\langle\mathbf{q}(\mathbf{x}, t, \theta), \mathbf{q}(\mathbf{x}, t, \theta)\rangle & =\sum_{i=1}^{P} \sum_{j=1}^{P} \mathbf{q}^{i}(\mathbf{x}, t) \mathbf{q}^{j}(\mathbf{x}, t)\left\langle\Psi_{i}(\theta), \Psi_{i}(\theta)\right\rangle \\
& =\sum_{i=1}^{P} \sum_{j=1}^{P} \mathbf{q}^{i}(\mathbf{x}, t) \mathbf{q}^{j}(\mathbf{x}, t) i ! \delta_{i j}=\sum_{i=1}^{P} i ! \mathbf{q}^{i}(\mathbf{x}, t)^{2} .
\end{aligned}
$$

The variance of the solution is then 


$$
\begin{aligned}
\text { var } & =\langle\mathbf{q}(\mathbf{x}, t, \theta), \mathbf{q}(\mathbf{x}, t, \theta)\rangle-\langle\mathbf{q}(\mathbf{x}, t, \theta), 1\rangle^{2} \\
& =\sum_{i=1}^{P} i ! \mathbf{q}^{i}(\mathbf{x}, t)^{2}-\mathbf{q}^{1}(\mathbf{x}, t)^{2}=\sum_{i=2}^{P} i ! \mathbf{q}^{i}(\mathbf{x}, t)^{2} .
\end{aligned}
$$

Stochastic collocation method. We now assume that the solution $\mathbf{q}(\mathbf{x}, t, \theta)$ is available in the form (4.12). First, we start by recalling that we have chosen to use the Gauss-Hermite collocation points $\left\{\theta_{j}\right\}_{1 \leq j \leq P}$; i.e., we have a quadrature formula that is exact for all polynomial $f(x)$ of degree at most $2 P-1$,

$$
\int_{-\infty}^{+\infty} e^{-\theta^{2}} f(\theta) d \theta=\sum_{j=1}^{P} \omega_{j} f\left(\theta_{j}\right),
$$

where $\left\{\omega_{j}\right\}_{1 \leq j \leq P}$ are the integration weight [4]. This formula can be used to compute the average of $\mathbf{q}(\mathbf{x}, t, \theta)$ given by (4.12):

$$
\langle\mathbf{q}(\mathbf{x}, t, \theta)\rangle=\sum_{i=1}^{P} \mathbf{q}^{i}(\mathbf{x}, t) \int_{-\infty}^{+\infty} \frac{1}{\sqrt{2 \pi}} e^{-\frac{\theta^{2}}{2}} L_{i}(\theta) d \theta .
$$

After a simple change of variable and using (4.20), we get

$$
\langle\mathbf{q}(\mathbf{x}, t, \theta)\rangle=\sum_{i=1}^{P} \mathbf{q}^{i}(\mathbf{x}, t) \sum_{j=1}^{P} \frac{1}{\sqrt{\pi}} \omega_{j} L_{i}\left(\sqrt{2} \theta_{j}\right) .
$$

This equation shows why we have decided to define Lagrange polynomials based on the collocation points $\sqrt{2} \theta_{j}$ instead of $\theta_{j}$. Indeed, using the fact that $L_{i}\left(\sqrt{2} \theta_{j}\right)=\delta_{i j}$, the above equation simplifies as

$$
\langle\mathbf{q}(\mathbf{x}, t, \theta)\rangle=\sum_{j=1}^{P} \frac{1}{\sqrt{\pi}} \omega_{j} \mathbf{q}^{j}(\mathbf{x}, t) .
$$

The procedure to compute the variance is similar, leading to

$$
\text { var }=\sum_{j=1}^{P} \frac{1}{\sqrt{\pi}} \omega_{j}\left(\mathbf{q}^{j}(\mathbf{x}, t)\right)^{2}-\langle\mathbf{q}(\mathbf{x}, t, \theta)\rangle^{2} .
$$

It is important to emphasize that the simple, and most expensive, approach of using a tensor product grid of Gauss quadratures is only one among many alternatives. This is discussed in detail in [17], where special attention is given to methods of relevance to problems discussed here.

4.5.2. Statistics of nonlinear quantities. Often, however, we are interested in the statistics of some derived, possibly nonlinear, functional, $F(\mathbf{q})$ of $\mathbf{q}(\mathbf{x}, t, \theta)$, e.g., computation of the impact on the RCS of the uncertainty in the scattering problem.

Let us utilize the idea behind the collocation approach and write

$$
F(\mathbf{q}(\theta))=\sum_{j=1}^{P} F\left(\mathbf{q}\left(\theta_{j}\right)\right) L_{j}(\theta) .
$$


Thus, we simply need to evaluate the general functionals at the values of $\theta_{j}$. However, since we have already obtained full probabilistic information in the expansions, (4.7) or (4.12), we can use these results directly to obtain the required information and, thus, the probabilistic information on $F(\mathbf{q})$. All informations of interest, e.g., moments, can now be extracted from this in the same way as for the simple variables. Naturally, one can evaluate the integrals using a classic Monte Carlo approach. This can be done at little cost since it requires only evaluation of the expansions and not solution of Maxwell's equations.

For the Galerkin method, one just needs an extra step to express the fields from a modal polynomial basis (4.7) to a nodal polynomial basis (4.12), where the coefficients $\mathbf{q}^{j}(\mathbf{x}, t)$ are computed by simple evaluation of $\mathbf{q}^{j}(\mathbf{x}, t)=\sum_{i=1}^{P} \mathbf{q}^{i}(\mathbf{x}, t) \Psi_{i}\left(\sqrt{2} \theta_{j}\right)$. Then, the procedure is the same as that for the collocation method.

5. Numerical examples. In the following we shall discuss a few examples used to validate the approach discussed above. These results are chosen largely to be simple enough to enable rigorous testing as well as to expose the generality and strength of the proposed technique.

5.1. One-dimensional material loaded cavity. As a first simple test case, we consider a one-dimensional PEC cavity loaded with two media, with a material interface at $x^{\text {mat }}=0$ and perfectly conducting walls at $x_{1}^{p e c}=-L$ and $x_{2}^{p e c}=L$, as shown in Figure 5.1. The aim of the test is to compute the first few resonance frequencies of the cavity. For the simple case considered here, these frequencies are given as

$$
\sqrt{\epsilon_{1}} \tan \left(\omega \sqrt{\epsilon_{2}}\left(L-x^{m a t}\right)\right)=-\sqrt{\epsilon_{2}} \tan \left(\omega \sqrt{\epsilon_{1}}\left(L+x^{m a t}\right)\right),
$$

where $\epsilon_{k}$ reflects the two different permittivities. We assume for simplicity that the materials are nonmagnetic, i.e., $\mu_{k}=1$, and solve on each domain $D_{k}$ the equations

$$
Q \frac{\partial \mathbf{q}}{\partial t}+\frac{\partial}{\partial x} \mathbf{F}(\mathbf{q})=0
$$

where

$$
\mathbf{q}=\left(\begin{array}{c}
\mathbf{E}_{k}^{y} \\
\mathbf{H}_{k}^{z}
\end{array}\right), \quad \mathbf{F}(\mathbf{q})=\left(\begin{array}{c}
\mathbf{H}_{k}^{z} \\
\mathbf{E}_{k}^{y}
\end{array}\right), \quad Q=\left(\begin{array}{cc}
\epsilon_{k} & 0 \\
0 & \mu_{k}
\end{array}\right)
$$

To compute the resonant frequencies, we solve the one-dimensional Maxwell's equations in the time-domain, subject to a broadband initial condition, and collect one or several time-traces at various points in the cavity. The spectrum of the time-series yields the resonant frequencies as strong peaks which are found automatically.

5.1.1. Uncertainty in material. In this first test we assume an uncertainty or randomness in the permittivity of the material in domain $D_{2}$ of the form

$$
\varepsilon(\mathbf{x}, \theta)= \begin{cases}1 & \text { if } \mathbf{x} \notin D_{2}, \\ 2.25\left(1+0.1 \frac{\theta^{2}}{1+\theta^{2}}\right) & \text { otherwise, }\end{cases}
$$

where, as everywhere else, $\theta$ is a Gaussian variable with zero mean and unit variance. This model ensures that the permittivity will remain positive in the domain $D_{2}$. Other parameters were fixed as $L=1$ and $\mu_{1}=\mu_{2}=1$. 


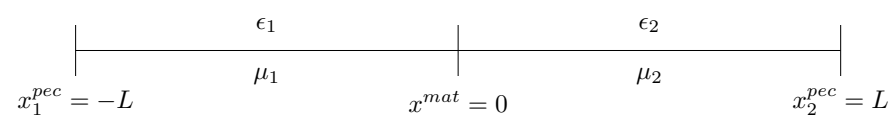

FIG. 5.1. Illustration of one-dimensional loaded cavity.

TABLE 5.1

Numerical mean and variance of resonance frequencies for cavity with random material. The discrepancy in the variance for $\omega_{1}$ is caused by quantization errors in the frequency identification approach and is not related to the accuracy of the modeling of the uncertainty. This is confirmed by having an identical error in the Monte Carlo model.

\begin{tabular}{|c|c|c|c|c|c|c|c|c|c|c|c|}
\hline \multicolumn{12}{|c|}{ Mean of Resonance Frequencies for Uncertainty in Material } \\
\hline \multicolumn{2}{|c|}{ iterations } & $\omega_{1}$ & $\omega_{2}$ & $\omega_{3}$ & $\omega_{4}$ & $\omega_{5}$ & $\omega_{6}$ & $\omega_{7}$ & $\omega_{8}$ & $\omega_{9}$ & $\omega_{10}$ \\
\hline \multirow{3}{*}{$\begin{array}{l}\text { Exact } \\
\text { Formula }\end{array}$} & 3600 & 1.1955 & 2.5689 & 3.6543 & 5.0158 & 6.2310 & 7.4079 & 8.7898 & 9.8795 & 11.2263 & 12.4614 \\
\hline & 7200 & 1.1957 & 2.5692 & 3.6549 & 5.0168 & 6.2319 & 7.4093 & 8.7913 & 9.8811 & 11.2285 & 12.4632 \\
\hline & 14400 & 1.1954 & 2.5688 & 3.6541 & 5.0155 & 6.2307 & 7.4074 & 8.7894 & 9.8790 & 11.2257 & 12.4609 \\
\hline \multirow{3}{*}{$\begin{array}{l}\text { Monte } \\
\text { Carlo }\end{array}$} & 300 & 1.1938 & 2.5715 & 3.6620 & 5.0209 & 6.2350 & 7.4083 & 8.7895 & 9.8837 & 11.2325 & 12.4675 \\
\hline & 600 & 1.1938 & 2.5712 & 3.6618 & 5.0230 & 6.2346 & 7.4102 & 8.7916 & 9.8869 & 11.2341 & 12.4680 \\
\hline & 1200 & 1.1938 & 2.5691 & 3.6583 & 5.0185 & 6.2301 & 7.4050 & 8.7862 & 9.8803 & 11.2262 & 12.4611 \\
\hline \multirow{3}{*}{$\begin{array}{c}\text { Stochastic } \\
\text { Galerkin } \\
P=40 \\
\end{array}$} & 3600 & 1.1938 & 2.5705 & 3.6595 & 5.0214 & 6.2313 & 7.4109 & 8.7936 & $\begin{array}{c}9.8836 \\
\end{array}$ & 11.2214 & 12.4753 \\
\hline & 7200 & $\begin{array}{l}1.1938 \\
\end{array}$ & 2.5708 & 3.6603 & 5.0225 & 6.2328 & 7.4126 & 8.7952 & 9.8842 & 11.2231 & 12.4775 \\
\hline & 14400 & 1.1938 & 2.5706 & 3.6594 & 5.0213 & 6.2316 & 7.4111 & 8.7937 & 9.8826 & 11.2203 & 12.4752 \\
\hline \multirow{3}{*}{$\begin{array}{c}\text { Stochastic } \\
\text { Galerkin } \\
P=100\end{array}$} & 3600 & 1.1938 & 2.5705 & 3.6592 & 5.0196 & 6.2330 & 7.4076 & 8.7901 & 9.8826 & 11.2291 & 12.4639 \\
\hline & 7200 & 1.1938 & 2.5707 & 3.6595 & 5.0199 & 6.2330 & 7.4074 & 8.7901 & 9.8828 & 11.2293 & 12.4645 \\
\hline & 14400 & 1.1938 & 2.5707 & 3.6599 & 5.0205 & 6.2334 & 7.4082 & 8.7908 & 9.8836 & 11.2300 & 12.4652 \\
\hline
\end{tabular}

\begin{tabular}{|c|c|c|c|c|c|c|c|c|c|c|c|}
\hline \multicolumn{12}{|c|}{ Variance of Resonance Frequencies for Uncertainty in Material } \\
\hline \multicolumn{2}{|c|}{ iterations } & $\omega_{1}$ & $\omega_{2}$ & $\omega_{3}$ & $\omega_{4}$ & $\omega_{5}$ & $\omega_{6}$ & $\omega_{7}$ & $\omega_{8}$ & $\omega_{9}$ & $\omega_{10}$ \\
\hline \multirow{3}{*}{$\begin{array}{c}\text { Exact } \\
\text { Formula }\end{array}$} & 3600 & $1.41 \mathrm{e}-04$ & $2.81 \mathrm{e}-04$ & $8.40 \mathrm{e}-04$ & $1.87 \mathrm{e}-03$ & $1.58 \mathrm{e}-03$ & $4.32 \mathrm{e}-03$ & $4.21 \mathrm{e}-03$ & $5.26 \mathrm{e}-03$ & $9.82 \mathrm{e}-03$ & $6.42 \mathrm{e}-03$ \\
\hline & 7200 & $1.38 \mathrm{e}-04$ & $2.75 \mathrm{e}-04$ & $8.23 \mathrm{e}-04$ & $1.83 \mathrm{e}-03$ & $1.54 \mathrm{e}-03$ & $4.23 \mathrm{e}-03$ & $4.11 \mathrm{e}-03$ & $5.15 \mathrm{e}-03$ & $9.60 \mathrm{e}-03$ & $6.28 \mathrm{e}-03$ \\
\hline & 14400 & $1.38 \mathrm{e}-04$ & $2.76 \mathrm{e}-04$ & $8.25 \mathrm{e}-04$ & $1.83 \mathrm{e}-03$ & $1.55 \mathrm{e}-03$ & $4.24 \mathrm{e}-03$ & $4.13 \mathrm{e}-03$ & $5.16 \mathrm{e}-03$ & $9.64 \mathrm{e}-03$ & $6.30 \mathrm{e}-03$ \\
\hline \multirow{3}{*}{$\begin{array}{l}\text { Monte } \\
\text { Carlo }\end{array}$} & 300 & $2.62 \mathrm{e}-29$ & $2.69 \mathrm{e}-04$ & $1.28 \mathrm{e}-03$ & $2.41 \mathrm{e}-03$ & $1.98 \mathrm{e}-03$ & $4.03 \mathrm{e}-03$ & $4.06 \mathrm{e}-03$ & $5.72 \mathrm{e}-03$ & $9.89 \mathrm{e}-03$ & $\begin{array}{l}7.04 \mathrm{e}-03 \\
\end{array}$ \\
\hline & 600 & $2.15 \mathrm{e}-28$ & $2.85 \mathrm{e}-04$ & $1.36 \mathrm{e}-03$ & $2.43 \mathrm{e}-03$ & $2.09 \mathrm{e}-03$ & $4.06 \mathrm{e}-03$ & $4.13 \mathrm{e}-03$ & $5.65 \mathrm{e}-03$ & $9.92 \mathrm{e}-03$ & $7.02 \mathrm{e}-03$ \\
\hline & 1200 & $1.66 \mathrm{e}-28$ & $3.92 \mathrm{e}-04$ & $1.49 \mathrm{e}-03$ & $2.44 \mathrm{e}-03$ & $2.30 \mathrm{e}-03$ & $4.47 \mathrm{e}-03$ & $4.52 \mathrm{e}-03$ & $6.13 \mathrm{e}-03$ & $1.08 \mathrm{e}-02$ & $7.46 \mathrm{e}-03$ \\
\hline \multirow{3}{*}{$\begin{array}{c}\text { Stochastic } \\
\text { Galerkin } \\
P=40\end{array}$} & $\overline{3600}$ & $4.06 \mathrm{e}-27$ & 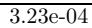 & $\bar{~} 1.43 \mathrm{e}-03$ & $\overline{2.40 \mathrm{e}-03}$ & $\begin{array}{l}2.18 \mathrm{e}-03 \\
\end{array}$ & $\overline{3.84 \mathrm{e}-03}$ & $\begin{array}{l}3.78 \mathrm{e}-03 \\
\end{array}$ & $\overline{5.62 \mathrm{e}-03}$ & $\begin{array}{c}9.76 \mathrm{e}-03 \\
\end{array}$ & $\begin{array}{l}7.66 \mathrm{e}-03 \\
\end{array}$ \\
\hline & 7200 & $5.84 \mathrm{e}-27$ & $3.06 \mathrm{e}-04$ & $1.42 \mathrm{e}-03$ & $2.32 \mathrm{e}-03$ & $2.18 \mathrm{e}-03$ & $3.77 \mathrm{e}-03$ & $3.72 \mathrm{e}-03$ & $5.45 \mathrm{e}-03$ & $9.62 \mathrm{e}-03$ & $7.58 \mathrm{e}-03$ \\
\hline & 14400 & $2.74 \mathrm{e}-07$ & $3.17 \mathrm{e}-04$ & $1.41 \mathrm{e}-03$ & $2.32 \mathrm{e}-03$ & $2.18 \mathrm{e}-03$ & $3.78 \mathrm{e}-03$ & $3.72 \mathrm{e}-03$ & $5.53 \mathrm{e}-03$ & $9.61 \mathrm{e}-03$ & $7.68 \mathrm{e}-03$ \\
\hline \multirow{3}{*}{$\begin{array}{c}\text { Stochastic } \\
\text { Galerkin } \\
P=100\end{array}$} & $\overline{3600}$ & $4.06 \mathrm{e}-27$ & $\overline{3.19 \mathrm{e}-04}$ & $\overline{1.42 \mathrm{e}-03}$ & $\overline{2.35 \mathrm{e}-03}$ & $\begin{array}{l}2.12 \mathrm{e}-03 \\
\end{array}$ & $4.23 \mathrm{e}-03$ & $4.19 \mathrm{e}-03$ & $\overline{5.86 \mathrm{e}-03}$ & $\begin{array}{l}1.03 \mathrm{e}-02 \\
\end{array}$ & $6.82 \mathrm{e}-03$ \\
\hline & 7200 & $5.84 \mathrm{e}-27$ & $3.10 \mathrm{e}-04$ & $1.41 \mathrm{e}-03$ & $2.38 \mathrm{e}-03$ & $2.16 \mathrm{e}-03$ & $4.29 \mathrm{e}-03$ & $4.24 \mathrm{e}-03$ & $5.89 \mathrm{e}-03$ & $1.06 \mathrm{e}-02$ & $7.12 \mathrm{e}-03$ \\
\hline & 14400 & $6.82 \mathrm{e}-27$ & $3.11 \mathrm{e}-04$ & $1.42 \mathrm{e}-03$ & $2.38 \mathrm{e}-03$ & $2.13 \mathrm{e}-03$ & $4.26 \mathrm{e}-03$ & $4.21 \mathrm{e}-03$ & $5.89 \mathrm{e}-03$ & $1.02 \mathrm{e}-02$ & $7.07 \mathrm{e}-03$ \\
\hline
\end{tabular}

For the stochastic Galerkin method with random permittivity, the terms $Q, Z, \bar{Z}$, $Y, \bar{Y}$ in the semidiscrete equation are all dependent on $\varepsilon(\mathbf{x}, \theta)$ so that the equations are given by (4.9). The case of random permittivity gives rise to a coupled system of $P$ deterministic equations, where the coupling is through the many scalar products defined in (4.9). These scalar products are computed in the preprocessing stage and stored.

Numerical results. We evaluate the technique by computing the mean and variance of the resonance frequencies of the loaded cavity. In Table 5.1 we show values computed by three different methods. The results labeled "Exact Formula" are obtained by performing Monte Carlo sampling on the equation that describes the resonance frequencies (5.1). As a benchmark, we show results obtained using a standard Monte Carlo method on (5.2) with up to 1200 samples. The frequencies in the stochastic Galerkin method are computed using the method described in section 4.5.1. Results for two values of $P, 40$ and 100, are shown. The results show good agreement between the stochastic Galerkin approach and the statistics of the exact solution as well as the Monte Carlo method, although it is not clear that the latter results are 
converged. The tables also show that while increasing $P$ does increase the accuracy of the data, the gain in accuracy is not significant. Note that the iteration in the Monte Carlo solutions amounts to actually solving the Maxwell's equations, i.e., the computational time scales with the number of iterations. For the chaos expansion method, the computational work scales with $P$ as the iterations simply amount to summing the final chaos expansions. Thus, the cost of the Monte Carlo approach is many times larger than the cost of using the techniques discussed here.

5.1.2. Uncertainty in boundary position. Let us now consider the case when the length of the cavity is unknown, i.e., when there is uncertainty associated with the position of the boundary. We choose to have uncertainty in the right boundary position so that the domain $D_{2}$ is of variable length. The position of the right boundary is assumed to be $x_{2}^{p e c}=L+g(\theta)$, where

$$
g(\theta)=0.1\left(\frac{\theta}{1+\theta^{2}}\right)
$$

All other parameters are fixed as $L=1, \mu_{1}=\mu_{2}=1, \epsilon_{1}=1$, and $\epsilon_{2}=2.25$.

For the stochastic Galerkin method with a random boundary position, we introduce a linear mapping from the variable $x$ into the new variable $\xi \in[-1,1]$, i.e., it is of fixed length. The mapping of the variable domain $D_{2}$ into a fixed domain of length $L$ is

$$
\xi=x^{m a t}+\left(L-x^{m a t}\right) \frac{x-x^{m a t}}{\left(L+g(\theta)-x^{m a t}\right)} .
$$

We can then express (5.2) in the new variable $\xi$ by

$$
Q \frac{\partial \mathbf{q}}{\partial t}+\frac{\partial \xi}{\partial x} \frac{\partial \mathbf{F}(\mathbf{q})}{\partial \xi}=0
$$

which is stated in a fixed computational domain. Note that this transformation should also be applied to the initial conditions. As the equations have changed only by the multiplication of the additional term $\frac{\partial \xi}{\partial x}$, and we now have a deterministic $Q$, the computational scheme is expressed as

$$
\begin{aligned}
& \forall k \in[1, P]: Q M k ! \frac{d \mathbf{q}_{N}^{k}}{d t}+S \sum_{i=1}^{P}\left\langle\frac{\partial \xi}{\partial x} \Psi_{i}, \Psi_{k}\right\rangle \cdot \mathbf{F}_{N}^{i}=F \sum_{i=1}^{P} \hat{\mathbf{n}} \cdot\left[\mathbf{F}_{N}^{i}-\mathbf{F}^{i *}\right] \\
& \hat{\mathbf{n}} \cdot\left[\mathbf{F}_{N}^{i}-\mathbf{F}^{i *}\right]=\left\langle\frac{\partial \xi}{\partial \mathbf{x}} \Psi_{i}, \Psi_{k}\right\rangle\left(\begin{array}{l}
\bar{Z}^{-1} \hat{\mathbf{n}} \times \hat{\mathbf{n}} \times\left[\mathbf{E}^{i}\right]-Z^{+} \bar{Z}^{-1} \hat{\mathbf{n}} \times\left[\mathbf{H}^{i}\right] \\
\bar{Y}^{-1} \hat{\mathbf{n}} \times \hat{\mathbf{n}} \times\left[\mathbf{H}^{i}\right]+Y^{+} \bar{Y}^{-1} \hat{\mathbf{n}} \times\left[\mathbf{E}^{i}\right]
\end{array}\right) .
\end{aligned}
$$

Thus, the random domain problem has been transformed into a random coefficient problem similar to the one discussed previously. Note that we apply this method in both domains, although in domain $D_{1}$, which is already of fixed length, the value of $\frac{\partial \xi}{\partial \mathbf{x}}$ is one.

Numerical results. We compute again the mean and variance of the resonance frequencies of the loaded cavity. Since the boundary moves only in a small region of the domain $D_{2}=\left[x^{m a t}, x_{2}^{p e c}\right]$, we split the domain $D_{2}$ into two artificial subdomains $D_{21}=\left[x^{\text {mat }}, x^{\text {split }}\right], D_{22}=\left[x^{\text {split }}, x_{2}^{\text {pec }}\right]$ such that $D_{2}=D_{21} \cup D_{22}$ and $x^{\text {split }}$ is chosen depending on how far the boundary can move inward. Then we can apply the 
TABLE 5.2

Numerical mean and variance of resonance frequencies for a cavity with an uncertain boundary.

\begin{tabular}{|c|c|c|c|c|c|c|c|c|c|c|c|}
\hline \multicolumn{10}{|c|}{ Mean of Resonance Frequencies for Uncertainty in Boundary Position } \\
\hline iterations & $\omega_{1}$ & $\omega_{2}$ & $\omega_{3}$ & $\omega_{4}$ & $\omega_{5}$ & $\omega_{6}$ & $\omega_{7}$ & $\omega_{8}$ & $\omega_{9}$ & $\omega_{10}$ \\
\hline Exact & 3600 & 1.2111 & 2.5906 & 3.6943 & 5.0714 & 6.2847 & 7.4973 & 8.8684 & 9.9860 & 11.3513 & 12.5698 \\
\cline { 2 - 14 } Formula & 7200 & 1.2111 & 2.5904 & 3.6941 & 5.0712 & 6.2844 & 7.4971 & 8.8678 & 9.9857 & 11.3511 & 12.5695 \\
\cline { 2 - 13 } & 14400 & 1.2111 & 2.5904 & 3.6941 & 5.0712 & 6.2843 & 7.4969 & 8.8679 & 9.9854 & 11.3508 & 12.5691 \\
\hline \hline \multirow{2}{*}{ Stochastic } & 3600 & 1.2199 & 2.5830 & 3.6943 & 5.0654 & 6.2871 & 7.4874 & 8.8631 & 9.9898 & 11.3518 & 12.5519 \\
Galerkin & 7200 & 1.2203 & 2.5834 & 3.6947 & 5.0652 & 6.2876 & 7.4881 & 8.8638 & 9.9903 & 11.3523 & 12.5534 \\
\cline { 2 - 12 }$P=40$ & 14400 & 1.2205 & 2.5832 & 3.6942 & 5.0644 & 6.2874 & 7.4883 & 8.8637 & 9.9900 & 11.3514 & 12.5529 \\
\hline \hline \multirow{2}{*}{ Stochastic } & 3600 & 1.2207 & 2.5820 & 3.6900 & 5.0693 & 6.2894 & 7.4934 & 8.8623 & 9.9982 & 11.3619 & 12.5679 \\
\cline { 2 - 12 } Galerkin & 7200 & 1.2205 & 2.5820 & 3.6896 & 5.0682 & 6.2886 & 7.4915 & 8.8606 & 9.9965 & 11.3597 & 12.5661 \\
$P=100$ & 14400 & 1.2210 & 2.5821 & 3.6899 & 5.0691 & 6.2895 & 7.4930 & 8.8620 & 9.9981 & 11.3615 & 12.5675 \\
\hline
\end{tabular}

\begin{tabular}{|c|c|c|c|c|c|c|c|c|c|c|c|}
\hline \multicolumn{12}{|c|}{ Variance of Resonance Frequencies for Uncertainty in Boundary Position } \\
\hline \multicolumn{2}{|c|}{ iterations } & $\omega_{1}$ & $\omega_{2}$ & $\omega_{3}$ & $\omega_{4}$ & $\omega_{5}$ & $\omega_{6}$ & $\omega_{7}$ & $\omega_{8}$ & $\omega_{9}$ & $\omega_{10}$ \\
\hline \multirow{3}{*}{$\begin{array}{c}\text { Exact } \\
\text { Formula }\end{array}$} & 3600 & $2.39 \mathrm{e}-04$ & $1.90 \mathrm{e}-03$ & $3.88 \mathrm{e}-03$ & $4.20 \mathrm{e}-03$ & $1.53 \mathrm{e}-02$ & $9.28 \mathrm{e}-03$ & $2.22 \mathrm{e}-02$ & $2.84 \mathrm{e}-02$ & $2.27 \mathrm{e}-02$ & $6.07 \mathrm{e}-02$ \\
\hline & 7200 & $2.43 \mathrm{e}-04$ & $1.94 \mathrm{e}-03$ & $3.95 \mathrm{e}-03$ & $4.27 \mathrm{e}-03$ & $1.55 \mathrm{e}-02$ & $9.44 \mathrm{e}-03$ & $2.25 \mathrm{e}-02$ & $2.88 \mathrm{e}-02$ & $2.37 \mathrm{e}-02$ & $6.22 \mathrm{e}-02$ \\
\hline & 14400 & $2.39 \mathrm{e}-04$ & $1.90 \mathrm{e}-03$ & $3.88 \mathrm{e}-03$ & $4.20 \mathrm{e}-03$ & $1.52 \mathrm{e}-02$ & $9.27 \mathrm{e}-03$ & $2.22 \mathrm{e}-02$ & $2.83 \mathrm{e}-02$ & $2.29 \mathrm{e}-02$ & $6.08 \mathrm{e}-02$ \\
\hline \multirow{3}{*}{$\begin{array}{c}\text { Stochastic } \\
\text { Galerkin } \\
P=40 \\
\end{array}$} & 3600 & 9.59 & 2.89 & $\overline{77.5}$ & $\overline{1.90}$ & 1.25 & 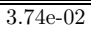 & 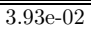 & & & 6.92 \\
\hline & 7200 & $9.63 \mathrm{e}-04$ & $2.90 \mathrm{e}-03$ & $7.64 \mathrm{e}-03$ & $1.91 \mathrm{e}-02$ & $1.25 \mathrm{e}-02$ & $3.81 \mathrm{e}-02$ & $3.97 \mathrm{e}-02$ & $5.08 \mathrm{e}-02$ & $8.97 \mathrm{e}-02$ & $6.98 \mathrm{e}-02$ \\
\hline & 14400 & $9.65 \mathrm{e}-04$ & $2.93 \mathrm{e}-03$ & $7.66 \mathrm{e}-03$ & $1.93 \mathrm{e}-02$ & $1.26 \mathrm{e}-02$ & $3.85 \mathrm{e}-02$ & $4.01 \mathrm{e}-02$ & $5.12 \mathrm{e}-02$ & $9.07 \mathrm{e}-02$ & $7.01 \mathrm{e}-02$ \\
\hline \multirow{3}{*}{$\begin{array}{c}\text { Stochastic } \\
\text { Galerkin } \\
P=100\end{array}$} & 3600 & 4 & & & $1.85 \mathrm{e}-02$ & $1.41 \mathrm{e}-02$ & $3.84 \mathrm{e}$ & $16 \mathrm{e}-02$ & & & 02 \\
\hline & 7200 & & & & & 1.4 & 3.8 & $4.12 \mathrm{e}-02$ & -02 & 9.0 & $6.21 \mathrm{e}-02$ \\
\hline & 14400 & $9.69 \mathrm{e}-04$ & $3.18 \mathrm{e}-03$ & $7.78 \mathrm{e}-03$ & $1.85 \mathrm{e}-02$ & $1.42 \mathrm{e}-02$ & $3.84 \mathrm{e}-02$ & $4.16 \mathrm{e}-02$ & $5.00 \mathrm{e}-02$ & $9.05 \mathrm{e}-02$ & $6.27 \mathrm{e}-02$ \\
\hline
\end{tabular}

transformation described in the previous section only to the subdomain $D_{22}$. This matches the physical properties of the case of an uncertain boundary more closely than mapping the entire domain $D_{2}$.

Table 5.2 shows statistics of the resonance frequencies computed by the stochastic Galerkin method and sampling of the exact solution. Since the Monte Carlo method is computationally far more expensive, we do not repeat the tests for all cases. The results again indicate that the stochastic Galerkin approach is in excellent agreement with the exact solution.

5.1.3. Uncertainty in the interface position. In this last example, we focus on the cavity where the position of an interface is uncertain, such that both domains are of variable length. We define the position of the material interface as $x^{\text {mat }}=$ $0+g(\theta)$, where

$$
g(\theta)=0.1\left(\frac{\theta}{1+\theta^{2}}\right)
$$

This allows the material interface to be positioned on both sides of the mean position. We fix other parameters as $L=1, \mu_{1}=\mu_{2}=1, \epsilon_{1}=1$, and $\epsilon_{2}=2.25$.

For the stochastic Galerkin method with random position of the interface, we proceed in a manner similar to that for the random boundary case and introduce a mapping of both domains from the variable $x$ to the new variable $\xi$. The mapping of the variable domains $D_{1}$ and $D_{2}$ into domains of fixed length $L$ is defined as

$$
\xi= \begin{cases}-L+L \frac{(x+L)}{(g(\theta)+L)} & \text { if } x \in D_{1}, \\ L \frac{(x-g(\theta))}{(L-g(\theta))} & \text { if } x \in D_{2} .\end{cases}
$$

The rest follows from (5.7)-(5.9). 
TABLE 5.3

Numerical mean and variance of resonance frequencies for cavity with an interface at random position.

\begin{tabular}{|c|c|c|c|c|c|c|c|c|c|c|c|}
\hline \multicolumn{10}{|c|}{ Mean of Resonance Frequencies for Uncertainty in Interface Position } \\
\hline iterations & $\omega_{1}$ & $\omega_{2}$ & $\omega_{3}$ & $\omega_{4}$ & $\omega_{5}$ & $\omega_{6}$ & $\omega_{7}$ & $\omega_{8}$ & $\omega_{9}$ & $\omega_{10}$ \\
\hline Exact & 3600 & 1.2115 & 2.5884 & 3.6975 & 5.0686 & 6.2832 & 7.5054 & 8.8486 & 10.0084 & 11.3353 & 12.5667 \\
\cline { 2 - 13 } Formula & 7200 & 1.2116 & 2.5885 & 3.6976 & 5.0691 & 6.2832 & 7.5063 & 8.8491 & 10.0089 & 11.3367 & 12.5672 \\
\cline { 2 - 13 } & 14400 & 1.2116 & 2.5886 & 3.6976 & 5.0689 & 6.2832 & 7.5063 & 8.8488 & 10.0093 & 11.3361 & 12.5671 \\
\hline \hline Stochastic & 3600 & 1.2166 & 2.5761 & 3.6902 & 5.0674 & 6.2832 & 7.5004 & 8.8567 & 10.0026 & 11.3383 & 12.5672 \\
Galerkin & 7200 & 1.2166 & 2.5761 & 3.6906 & 5.0682 & 6.2832 & 7.5015 & 8.8575 & 10.0030 & 11.3401 & 12.5677 \\
\cline { 2 - 12 }$P=40$ & 14400 & 1.2168 & 2.5761 & 3.6902 & 5.0679 & 6.2832 & 7.5015 & 8.8571 & 10.0032 & 11.3391 & 12.5672 \\
\hline \hline Stochastic & 3600 & 1.2172 & 2.5761 & 3.6903 & 5.0683 & 6.2832 & 7.5140 & 8.8631 & 10.0039 & 11.3378 & 12.5670 \\
Galerkin & 7200 & 1.2166 & 2.5761 & 3.6908 & 5.0672 & 6.2832 & 7.5122 & 8.8621 & 10.0026 & 11.3353 & 12.5678 \\
$P=100$ & 14400 & 1.2166 & 2.5761 & 3.6905 & 5.0674 & 6.2832 & 7.5120 & 8.8626 & 10.0030 & 11.3361 & 12.5675 \\
\hline
\end{tabular}

\begin{tabular}{|c|c|c|c|c|c|c|c|c|c|c|c|}
\hline \multicolumn{12}{|c|}{ nce of Resonance Frequencies for Uncertainty in Interface Position } \\
\hline \multicolumn{2}{|c|}{ iterations } & $\omega_{1}$ & $\omega_{2}$ & $\omega_{3}$ & $\omega_{4}$ & $\omega_{5}$ & $\omega_{6}$ & $\overline{\omega_{7}}$ & $\omega_{8}$ & $\omega_{9}$ & $\omega_{10}$ \\
\hline \multirow{3}{*}{$\begin{array}{l}\text { Exact } \\
\text { Formula }\end{array}$} & 3600 & $2.85 \mathrm{e}-04$ & $2.24 \mathrm{e}-04$ & $4.71 \mathrm{e}-04$ & $4.76 \mathrm{e}-03$ & $1.70 \mathrm{e}-05$ & $1.01 \mathrm{e}-02$ & $2.94 \mathrm{e}-03$ & $4.05 \mathrm{e}-03$ & $2.02 \mathrm{e}-02$ & $9.08 \mathrm{e}-04$ \\
\hline & 7200 & $2.84 \mathrm{e}-04$ & $2.23 \mathrm{e}-04$ & $4.70 \mathrm{e}-04$ & $4.75 \mathrm{e}-03$ & $1.69 \mathrm{e}-05$ & $1.04 \mathrm{e}-02$ & $3.13 \mathrm{e}-03$ & $4.34 \mathrm{e}-03$ & $2.06 \mathrm{e}-02$ & $1.34 \mathrm{e}-03$ \\
\hline & 14400 & $2.88 \mathrm{e}-04$ & $2.26 \mathrm{e}-04$ & $4.76 \mathrm{e}-04$ & $4.82 \mathrm{e}-03$ & $1.73 \mathrm{e}-05$ & $1.06 \mathrm{e}-02$ & $3.27 \mathrm{e}-03$ & $4.53 \mathrm{e}-03$ & $2.09 \mathrm{e}-02$ & $1.41 \mathrm{e}-03$ \\
\hline \multirow{3}{*}{$\begin{array}{c}\text { Stochastic } \\
\text { Galerkin } \\
P=40\end{array}$} & $\overline{3600}$ & .12 & $3.65 \mathrm{e}-28$ & 77.7 & $\overline{5.83 \mathrm{e}-03}$ & $1.54 \mathrm{e}-25$ & $\overline{9.08 \mathrm{e}-03}$ & $\begin{array}{c}3.74 \mathrm{e}-03 \\
\end{array}$ & 5.50 & $\overline{2.03}$ & $\overline{2.13}$ \\
\hline & 7200 & 9.13 & e- 26 & 7.6 & 5.7 & $2.27 \mathrm{e}-25$ & 9.14 & $3.73 \mathrm{e}-03$ & 5.5 & 2.0 & 2.38 \\
\hline & 14400 & $9.16 \mathrm{e}-04$ & $6.90 \mathrm{e}-27$ & $7.75 \mathrm{e}-04$ & $5.90 \mathrm{e}-03$ & $7.78 \mathrm{e}-26$ & $9.14 \mathrm{e}-03$ & $3.73 \mathrm{e}-03$ & $5.56 \mathrm{e}-03$ & $2.05 \mathrm{e}-02$ & $2.21 \mathrm{e}-03$ \\
\hline \multirow{3}{*}{$\begin{array}{c}\text { Stochastic } \\
\text { Galerkin } \\
P=100\end{array}$} & $\overline{3600}$ & 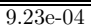 & (3.65e-28 & $=\overline{7.73 \mathrm{e}-04}$ & 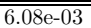 & $=1.54 \mathrm{e}-25$ & 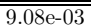 & (3.26e-03 & $\overline{\overline{5.91}}$ & 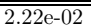 & $\overline{1.88 \mathrm{e}-03}$ \\
\hline & 7200 & $9.13 \mathrm{e}-04$ & $1.76 \mathrm{e}-26$ & $7.59 \mathrm{e}-04$ & $5.94 \mathrm{e}-03$ & $2.27 \mathrm{e}-25$ & $9.03 \mathrm{e}-03$ & $3.22 \mathrm{e}-03$ & $5.88 \mathrm{e}-03$ & $2.20 \mathrm{e}-02$ & $2.16 \mathrm{e}-03$ \\
\hline & 14400 & $9.12 \mathrm{e}-04$ & $6.90 \mathrm{e}-27$ & $7.67 \mathrm{e}-04$ & $5.96 \mathrm{e}-03$ & $7.78 \mathrm{e}-26$ & $8.92 \mathrm{e}-03$ & $3.20 \mathrm{e}-03$ & $5.84 \mathrm{e}-03$ & $2.17 \mathrm{e}-02$ & $2.28 \mathrm{e}-03$ \\
\hline
\end{tabular}

Numerical results. We split the domains into smaller regions, as described in the previous case, in order to match the physical properties of the cavity. In this case, the mapping is applied only to the two subdomains adjacent to the material interface. Table 5.3 shows statistics of the resonance frequencies computed by the stochastic Galerkin method and sampling of the exact solution. The quality of the results are similar to those of the previous test cases.

5.2. Two-dimensional circular cylinder. As a second and more advanced test, we consider plane wave scattering by a two-dimensional circular cylinder of radius unity. The measure of interest is the RCS, defined as

$$
R C S_{d b}(\phi)=10 \log \left(2 \pi \frac{|\mathbf{F}(\phi)|^{2}}{\left|\mathbf{E}_{i}\right|^{2}}\right),
$$

where $\mathbf{E}_{i}$ is the incident field and $\mathbf{F}(\phi)$ is a nonlinear function of $\mathbf{E}(\mathbf{x}, t, \theta)$ and $\mathbf{H}(\mathbf{x}, t, \theta)$, computing the scattered far field as a function of the polar angle, $\phi$. In this particular case, $\mathbf{F}(\phi)$ is the near-to-far-field transformation along some closed contour [15].

Typical meshes are represented in Figure 5.2, where the number of elements is 1141. The domain extends from -6 to +6 , and a perfectly matched layer (PML) of width 1 is used to truncate the domain (see [1] for details). Degree five polynomials are enough to ensure that convergence is achieved in the physical space, and six modes in the chaos expansion (or six nodes for the collocation formulation, i.e., $P=6$ ) are also enough for good convergence in probability space.

5.2.1. Uncertainty in the source term. Let us denote by $\Gamma$ the boundary of a PEC two-dimensional circular cylinder contained in the domain $\Omega$. In this first example, we consider the following model for the uncertainty in the source term appearing in (2.1): 


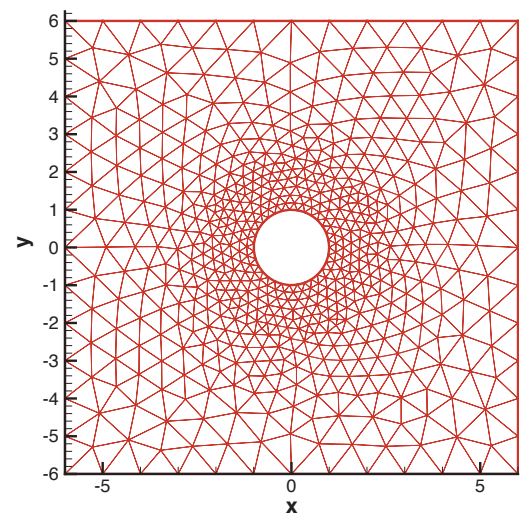

FIG. 5.2. Typical mesh using 1141 spectral elements.

$$
\mathbf{S}^{E}= \begin{cases}\mathbf{E}_{0} \sin (2 \pi \omega(\mathbf{k}(\theta) \cdot \mathbf{x}-t) & \text { if } \mathbf{x} \in \Gamma \\ \mathbf{0} & \text { otherwise }\end{cases}
$$

where $\mathbf{k}(\theta)$ is an uncertain wave vector. We assume that the value of the wave vector $\mathbf{k}(\theta)$ is subject to a small random perturbation from its deterministic value $\mathbf{k}_{0}=\left(r_{0} \cos \left(\alpha_{0}\right), r_{0} \sin \left(\alpha_{0}\right)\right)$. More precisely, we assume that this vector can vary in one of the following two ways:

1. Randomness in the direction of $\mathbf{k}$ :

$$
\mathbf{k}(\theta)=\left(\begin{array}{c}
r_{0} \cos \left(\alpha_{0}+0.1 \theta\right) \\
r_{0} \sin \left(\alpha_{0}+0.1 \theta\right)
\end{array}\right) \text {. }
$$

2. Randomness in the modulus of $\mathbf{k}$ :

$$
\mathbf{k}(\theta)=\left(\begin{array}{c}
r_{0}(1+0.1 \theta) \cos \left(\alpha_{0}\right) \\
r_{0}(1+0.1 \theta) \sin \left(\alpha_{0}\right)
\end{array}\right) \text {. }
$$

The latter case mimics a slight variation in the frequency of the source.

Stochastic Galerkin method. For the stochastic Galerkin method, the source term in the semidiscrete equation (4.9) becomes

$$
\mathbf{S}_{N}^{k}= \begin{cases}\mathbf{E}_{0}\left\langle\sin (2 \pi \omega(\mathbf{k}(\theta) \cdot \mathbf{x}-t)), \Psi_{k}\right\rangle & \text { if } \mathbf{x} \in \Gamma, \\ \mathbf{0} & \text { otherwise, }\end{cases}
$$

or, after a simple trigonometric manipulation,

$$
\mathbf{S}_{N}^{k}=\left\{\begin{array}{cl}
\mathbf{E}_{0}\left(\left\langle\sin (2 \pi \omega \mathbf{k}(\theta) \cdot \mathbf{x}), \Psi_{k}\right\rangle \cos (2 \pi \omega t)\right. & \\
\left.-\left\langle\cos (2 \pi \omega \mathbf{k}(\theta) \cdot \mathbf{x}), \Psi_{k}\right\rangle \sin (2 \pi \omega t)\right) & \text { if } \mathbf{x} \in \Gamma, \\
\mathbf{0} & \text { otherwise. }
\end{array}\right.
$$

The coefficients $\left\langle\sin (2 \pi \omega \mathbf{k}(\theta) \cdot \mathbf{x}), \Psi_{k}\right\rangle$ and $\left\langle\cos (2 \pi \omega \mathbf{k}(\theta) \cdot \mathbf{x}), \Psi_{k}\right\rangle$ in the above expression can be precomputed, stored, and then used to impose the boundary conditions at any subsequent time steps. Furthermore, by assuming that the permittivity $\epsilon$ and the permeability $\mu$ are deterministic, using the orthogonality of Hermite polynomials (4.5), equation (4.9) simplifies to

$$
\forall k \in[1, P]: k ! Q M \frac{d \mathbf{q}_{N}^{k}}{d t}+k ! S \cdot \mathbf{F}_{N}^{k}-M \mathbf{S}_{N}^{k}=F \hat{\mathbf{n}} \cdot\left[\mathbf{F}_{N}^{k}-\mathbf{F}^{k *}\right] .
$$



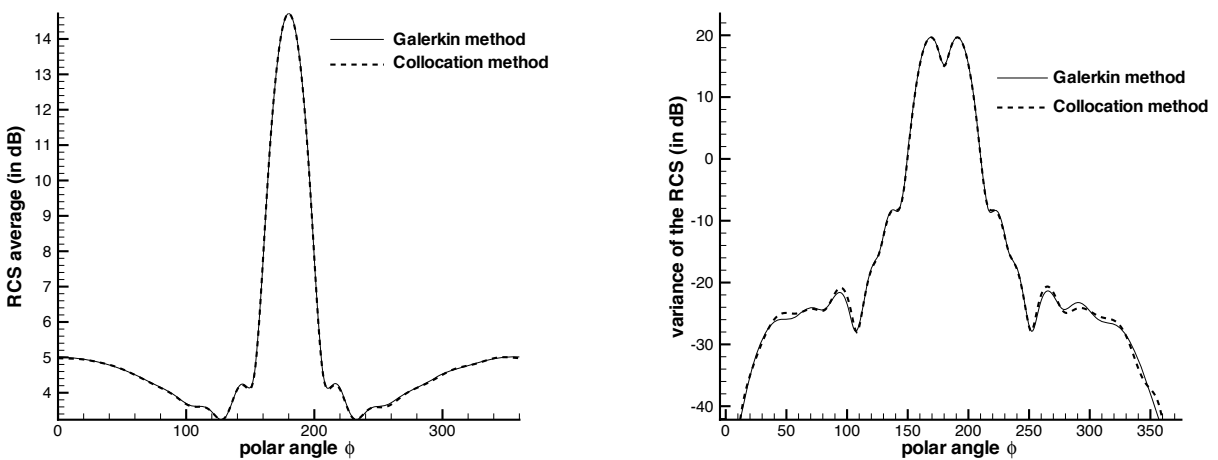

FIG. 5.3. Comparison of the stochastic Galerkin method and the stochastic collocation method. Left: Average of the RCS for the randomness in the angle of the wave. Right: Variance of the RCS for the randomness in the angle of the wave vector.

The above equation highlights that for this test case, all the modes are decoupled, and that the cost of solving it, compared to a deterministic simulation, is simply proportional to the number of modes in the chaos expansion. For this particular case, the costs of the Galerkin and collocation methods are the same.

Stochastic collocation method. For the stochastic collocation method, the source term in the semidiscrete equation (4.14) becomes

$$
\mathbf{S}_{N}^{j}= \begin{cases}\mathbf{E}_{0} \sin \left(2 \pi \omega\left(\mathbf{k}\left(\sqrt{2} \theta_{j}\right) \cdot \mathbf{x}-t\right)\right) & \text { if } \mathbf{x} \in \Gamma, \\ \mathbf{0} & \text { otherwise. }\end{cases}
$$

As always for the stochastic collocation technique, the nodal solutions $\mathbf{q}_{N}^{j}$ are decoupled from each other and are obtained by solving $P$ independent systems of the form

$$
\forall j \in[1, P]: Q M \frac{d \mathbf{q}_{N}^{j}}{d t}+S \cdot \mathbf{F}_{N}^{j}-M \mathbf{S}_{N}^{j}=F \hat{\mathbf{n}} \cdot\left[\mathbf{F}_{N}^{j}-\mathbf{F}^{j *}\right] .
$$

Numerical results. We conduct the first test to compare the results of the average and variance of the RCS computed by the stochastic collocation method and the stochastic Galerkin method. For the numerical experiments, we set $r_{0}=1$ and $\alpha_{0}=\pi$ in (5.14)-(5.15). Figure 5.3 shows the average and the variance of the RCS, and it can be seen that for the same discretization, they give identical results in agreement with the discussion in section 4.4. This case corresponds to uncertainty in the angle of the wave vector, and similar results are obtained for uncertainty in the norm of the wave vector. Figure 5.4 shows the average of the RCS and its possible variations for the randomness in the angle of $\mathbf{k}$ and in the modulus of $\mathbf{k}$. It appears that the uncertainty in the angle of $\mathbf{k}$ primarily affects the RCS in the forward scatter, whereas the uncertainty in the norm of $\mathbf{k}$ mainly affects the RCS in the sidebands.

5.2.2. Uncertainty in materials. We now assume that the permittivity inside a cylindrical domain $D$ is unknown, whereas the permittivity outside this domain is deterministic, e.g.,

$$
\varepsilon(\mathbf{x}, \theta)= \begin{cases}1 & \text { if } \mathbf{x} \notin D \\ 2.25 e^{0.1 \theta} & \text { otherwise }\end{cases}
$$



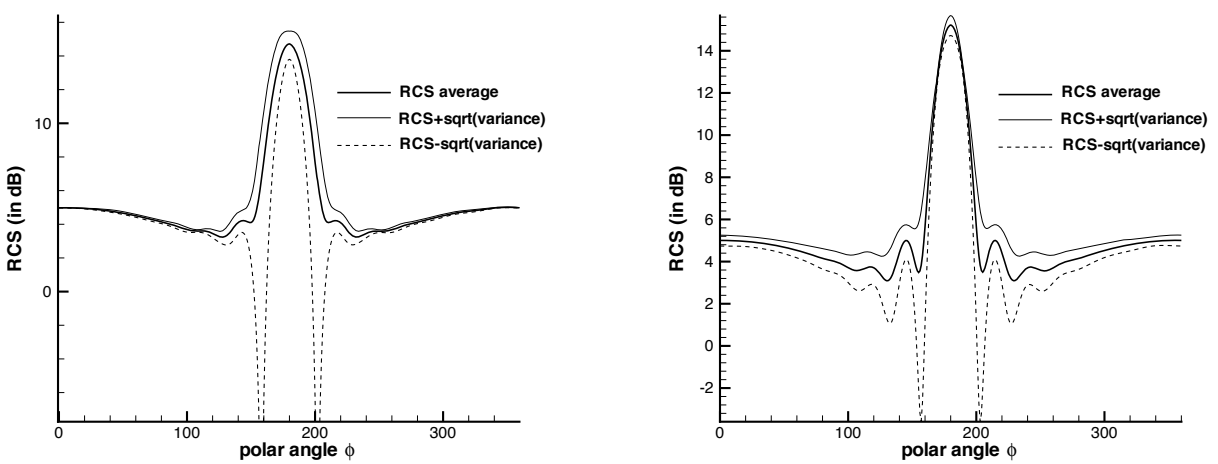

FIG. 5.4. Left: RCS for the randomness in the angle of the wave vector. Right: RCS for the randomness in the norm of the wave vector. Results are shown with the mean RCS as well as \pm 1 standard deviation.

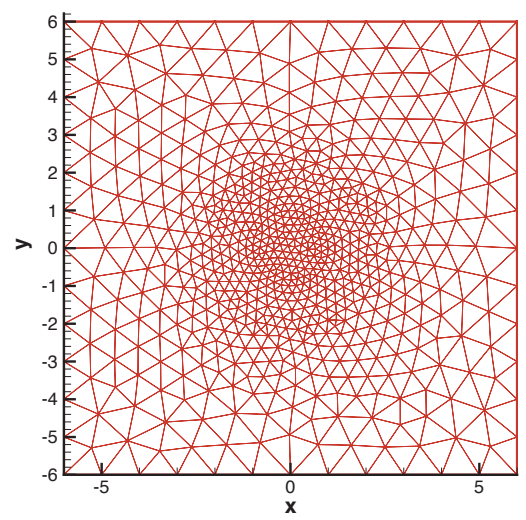

FIG. 5.5. Typical mesh using 1318 spectral elements.

Here again, our concern is not the correctness of the probabilistic law chosen in (5.21) for the uncertainty of the permittivity $\varepsilon(\mathbf{x}, \theta)$, since for any reasonable law, the techniques presented in this paper should work equally well. With an uncertainty of the form $e^{0.1 \theta}$ (which is a log-normal law), the permittivity is guaranteed to remain positive. For uncertainty in the permittivity of the material, the source term of (2.1) takes the form

$$
\mathbf{S}^{E}=-\left(\epsilon(\mathbf{x}, \theta)-\epsilon^{i}\right) \frac{\partial \mathbf{E}^{i}}{\partial t}
$$

where $\left(\mathbf{E}^{i}, \mathbf{H}^{i}\right)$ denotes the incident field which is a solution to Maxwell's equations. For that test case, it is necessary to mesh the entire domain, as shown in Figure 5.5 (here 1318 spectral elements are represented).

Stochastic Galerkin method. Projecting the source term using the chaos basis, we obtain

$$
\forall k \in[1, P]: \mathbf{S}_{N}^{k}=-\left(\left\langle\epsilon(\mathbf{x}, \theta), \Psi_{k}\right\rangle-\epsilon^{i}\right) \frac{\partial \mathbf{E}^{i}}{\partial t}
$$



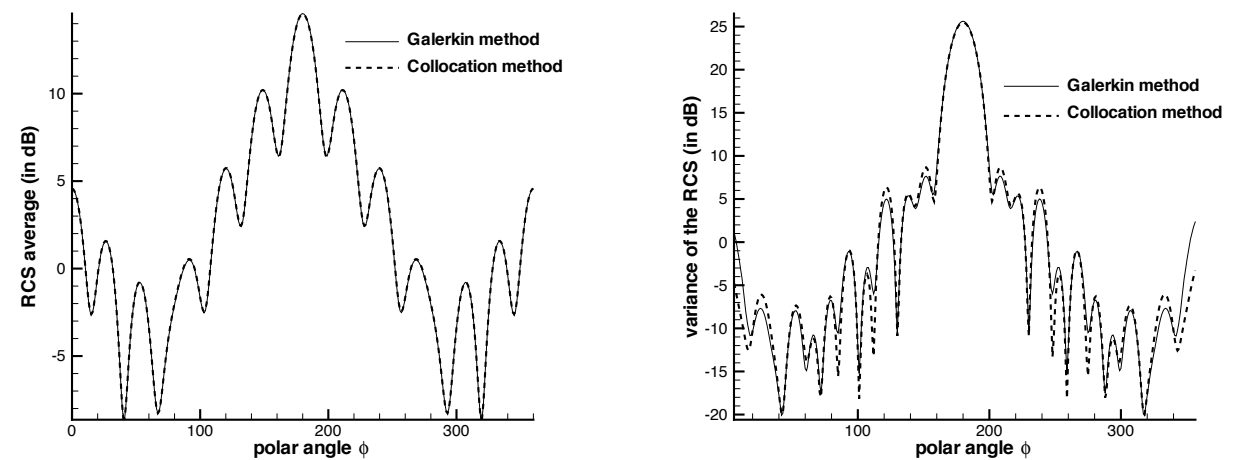

FIG. 5.6. Comparison of the stochastic Galerkin method and the stochastic collocation method. Left: Average of the RCS for the randomness in material. Right: Variance of the RCS for the randomness in material.

In this case, the equation to be solved is (4.9) and the source term is given by (5.23). Note that in this case, the size of the coupled system to be solved is $P$ times the size of the system coming from a deterministic simulation.

Stochastic collocation method. For the stochastic collocation method, the source term of (4.14) is simply

$$
\mathbf{S}_{N}^{j}=-\left(\epsilon\left(\mathbf{x}, \sqrt{2} \theta_{j}\right)-\epsilon^{i}\right) \frac{\partial \mathbf{E}^{i}}{\partial t} .
$$

In that case, the stochastic collocation method is cheaper than the stochastic Galerkin method since it only requires one to solve $P$ decoupled systems of the size of a deterministic problem.

Numerical results. Here we again compare the results of the RCS for both the stochastic collocation method and the stochastic Galerkin method. Figure 5.6 shows the average and the variance of the RCS. Although the results are not strictly identical, the small differences can be attributed to the nonsmooth nature of the RCS for this problem, which makes convergence more difficult to achieve. Figure 5.7 shows the average of the RCS and the possible variations around its average value. Note that for this test case, and contrary to the previous case, the whole RCS is affected by the uncertainty.

5.2.3. Uncertainty in shape. For this last example, the exact location of the boundary of an object is assumed to be unknown. One way to solve this problem is to generate different meshes for different radii of the cylinder and to perform a Monte Carlo simulation with those meshes. Since this cannot be achieved in an automatic and efficient way, in this paper we proceed differently, inspired by the one-dimensional approach based on mappings.

The idea is to keep the same mesh, the mean mesh, and to randomly move one side of the triangles sitting on the cylinder surface. For example, the triangle $\left(\mathbf{u}_{1}, \mathbf{u}_{2}, \mathbf{u}_{3}\right)$ has the side $\left(\mathbf{u}_{1}, \mathbf{u}_{3}\right)$ sitting on the cylinder surface (see Figure 5.8). For the deterministic case, the physical element is mapped into the parent element $\mathcal{T}$ and the transformation is written as

$$
\mathbf{x}(\xi, \eta)=(1-\xi-\eta) \mathbf{u}_{1}+\xi \mathbf{u}_{2}+\eta \mathbf{u}_{3} \text { for }(\xi, \eta) \in \mathcal{T} .
$$




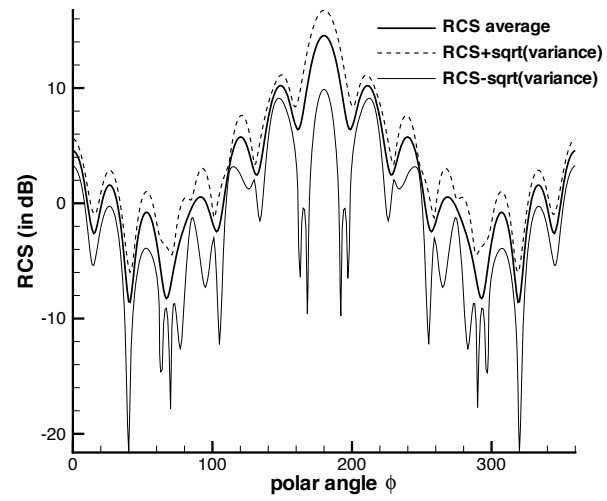

FIG. 5.7. RCS for uncertainty in the material. Results are shown with the mean RCS as well as \pm 1 standard deviation.

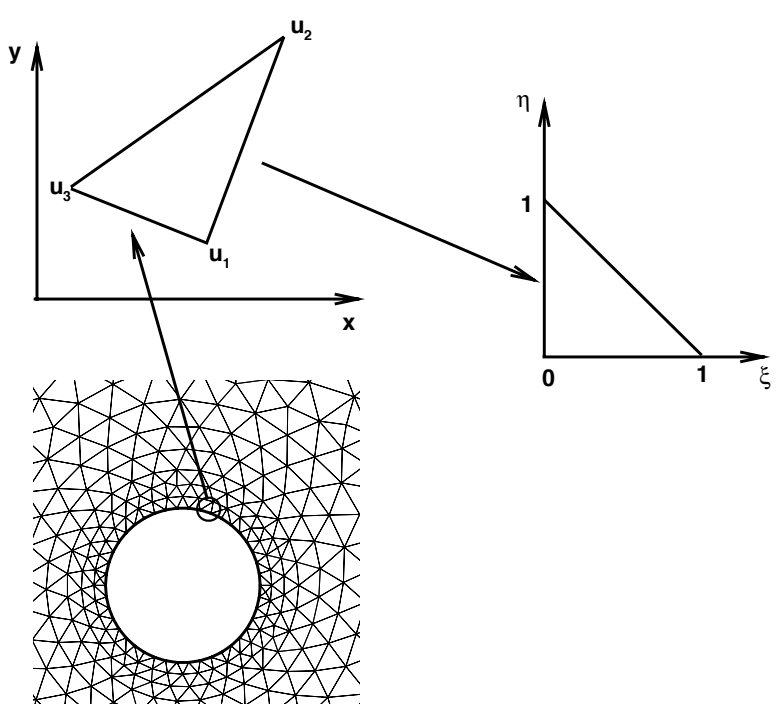

FIG. 5.8. Illustration for the randomness in shape.

Note that the Jacobian $J(\xi, \eta)=\left(\frac{\partial \mathbf{x}}{\partial \eta}, \frac{\partial \mathbf{x}}{\partial \xi}\right)^{T}$ of transformation (5.25) is only a function of $(\xi, \eta)$. Assuming randomness of the form $e^{0.1 \theta}$, the points $\mathbf{u}_{1}$ and $\mathbf{u}_{3}$ take a new position $e^{0.1 \theta} \mathbf{u}_{1}$ and $e^{0.1 \theta} \mathbf{u}_{3}$, respectively, and the mapping (5.25) becomes

$$
\mathbf{x}(\xi, \eta)=(1-\xi-\eta) e^{0.1 \theta} \mathbf{u}_{1}+\xi e^{0.1 \theta} \mathbf{u}_{2}+\eta \mathbf{u}_{3} \text { for }(\xi, \eta) \in \mathcal{T} .
$$

The Jacobian of transformation (5.26) is now a function of $(\xi, \eta, \theta)$. The determinant of the Jacobian, which appears as a multiplicative coefficient in Maxwell's equations, is random, and this makes Maxwell's equation a stochastic PDE, which can be solved with the techniques described above (collocation or Galerkin stochastic method). The advantage of the procedure lies in the fact that one need only generate a single mesh, the randomness being modeled by the Jacobian of the transformation. 

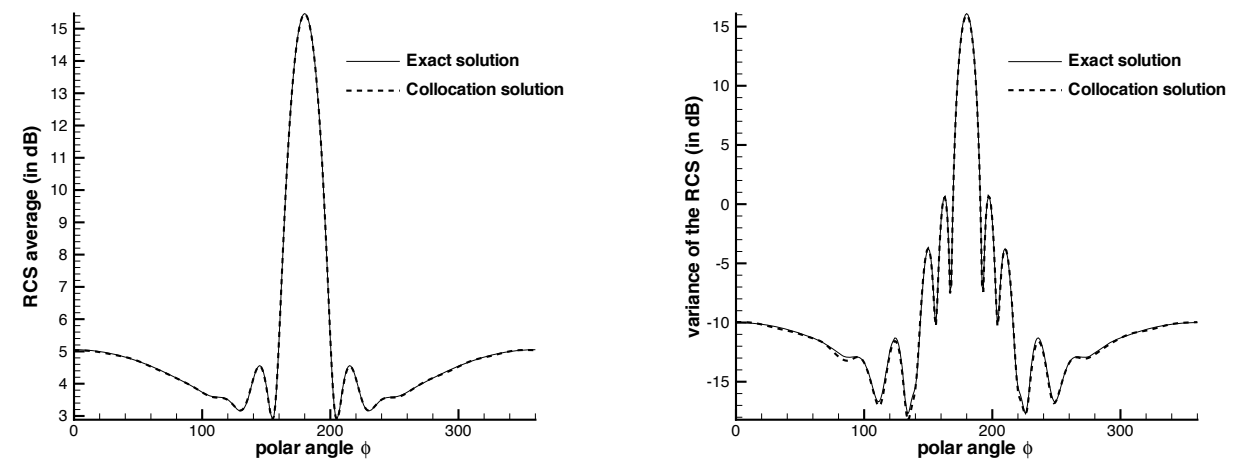

FIG. 5.9. Comparison of the stochastic collocation solution with the exact solution. Left: Average of the RCS for the uncertainty in shape. Right: Variance of the RCS for uncertainty in shape.

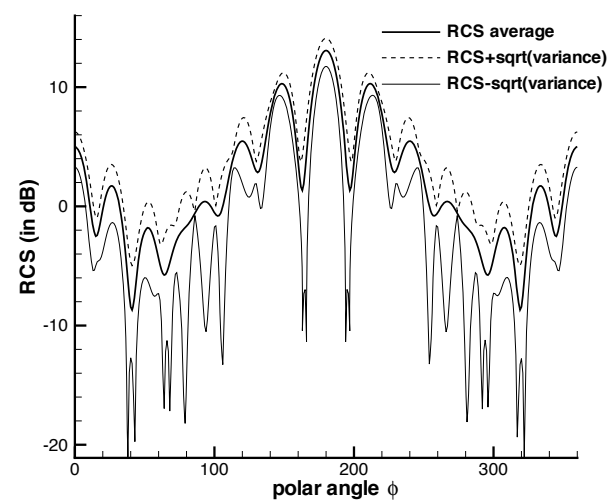

FIG. 5.10. RCS for material cylinder with uncertain radius. Results are shown with the mean $R C S$ as well as \pm 1 standard deviation.

Numerical results for a PEC cylinder with uncertain radius. We test the approach by considering a PEC cylinder with an uncertain radius, the randomness being described by (5.26). For the deterministic problem, an exact RCS is available [2]. Therefore, the coefficients in (5.3) can be computed exactly and an exact stochastic solution for the RCS is available for the random radius problem. This enables us to compare the statistics of the numerical stochastic collocation solution with the exact one. This is what is done in Figure 5.9, which represents the average and the variance of the RCS (the mesh used for the numerical results is the same as in the previous section). We can see that the two solutions match very well.

Numerical results for a cylindrical material with uncertain radius. For this last example, the exact radius of the cylindrical boundary of a cylinder with a permittivity $\epsilon_{1}$ is uncertain and is assumed to be described by (5.26). We also assume that outside the object, the relative permittivity is equal to one. Figure 5.10 shows the average of the RCS for this problem and the possible variations around its average value.

6. Concluding remarks. In this paper we discussed the use of probabilistic methods, rather than more traditional statistical methods such as Monte Carlo, to 
enable the computation of quantitative measures for the impact of uncertainties on computational results, exemplified by the solution of the time-domain Maxwell's equations. These uncertainties can be due to a lack of knowledge, or lack of control over variations, or can simply be used as a model to compute the mean and variances over an ensemble of data, e.g., to compute the RCS of a fleet of aircraft, all of the same type but with minor differences.

The approach, based on chaos expansions, has the following significant advantages:

- It is simple, systematic, and accurate, and can be used in connection with existing validated codes with only minimal changes.

- The representation of probability distributions, which are most often smooth, by using high-order polynomials can be expected to be close to optimal and leads to a need for very few terms, i.e., few samples, without sacrificing the accuracy.

- It enables the computation of an ensample of solutions in space and time with an associated probability measure. It can handle large degrees of uncertainty simply by increasing the length of the expansion.

- It is flexible enough to handle a large variety of different types of uncertainty, e.g., materials, initial conditions, and shapes.

The computational results validate these claims and show the potential of computing high-order moments, in particular variances/sensitivities, thus enabling the computation of numerical error bars.

Throughout this paper, the dimension of the random space $d$ is set to 1 , and for the stochastic collocation method, the computational cost is proportional to $P$, where $P$ is the number of collocation points. For more general problems with random dimensions $d>1$, the computational cost of the collocation method would be proportional to $P^{d}$ (for Galerkin polynomial chaos, it could be $P^{2 d}$, where, in that case, $P$ denotes the number of modes). This means that for problems where the correlation length is very small compared to the size of the problem, this method becomes more expensive, as a significant number of independent random variables is needed. For such cases requiring high-dimensional random spaces, the stochastic collocation methods discussed in [17] hold significant promise, as one can choose to limit the attention to high-dimensional integration formulas, thus dramatically reducing the number of samples even for high-dimensional randomness. As we have discussed in section 4.4, however, there is significant potential for reducing the computational cost by developing a theory that offers insight into how uncertainty can propagate through dynamical systems and what regularity one can expect for the underlying probability densities.

\section{REFERENCES}

[1] S. Abarbanel, D. Gottlieb, and J. S. Hesthaven, Long time behavior of the perfectly matched layer equations in computational electromagnetics, J. Sci. Comput., 17 (2002), pp. $405-422$.

[2] J. J. Bowman, T. B. A. Senior, and P. L. Ushlenghi, eds., Electromagnetic and Acoustic Scattering by Simple Shapes, North-Holland, Amsterdam, 1969.

[3] R. H. Cameron And W. T. Martin, The orthogonal development of nonlinear functionals in series of Fourier-Hermite functionals, Ann. of Math. (2), 48 (1947), pp. 385-392.

[4] C. Canuto, M. Y. Hussaini, A. Quarteroni, and T. A. Zang, Spectral Methods in Fluid Mechanics, Springer-Verlag, New York, 1988.

[5] Q. Y. Chen, D. Gottlieb, And J. S. Hesthaven, Uncertainty analysis for steady-state inviscid Burgers equation, J. Comput. Phys., 204 (2005), pp. 378-398. 
[6] D. Funaro, Polynomial Approximations of Differential Equations, Lecture Notes in Phys. New Ser. m Monogr. 8, Springer-Verlag, Berlin, 1992.

[7] R. G. Ghanem and P. Spanos, Stochastic Finite Elements: A Spectral Approach, SpringerVerlag, New York, 1991.

[8] D. Gottlieb and S. A. Orszag, Numerical Analysis of Spectral Methods: Theory and Applications, CBMS-NSF Regional Conf. Ser. in Appl. Math. 26, SIAM, Philadelphia, 1977.

[9] J. S. Hesthaven And T. Warburton, High-order nodal methods on unstructured grids. I. Time-domain solution of Maxwell's equations, J. Comput. Phys., 181 (2002), pp. 1-34.

[10] J. S. Hesthaven and T. WARBurton, High-order accurate methods for time-domain electromagnetics, CMES-Computer Modeling in Engineering and Sciences, 5 (2004), pp. 395-408.

[11] J. S. Hesthaven and T. WARburton, High-order nodal discontinuous Galerkin methods for the Maxwell eigenvalue problem, Philos. Trans. R. Soc. Lond. Ser. A Math. Phys. Eng. Sci., 362 (2004), pp. 493-524.

[12] J. S. Hesthaven and T. Warburton, Discontinuous Galerkin methods for the time-domain Maxwell's equations: An introduction, ACES Newsletter, 19 (2004), pp. 10-29.

[13] M. E. J. Newman and G. T. Barkema, Monte Carlo Methods in Statistical Physics, Oxford University Press, Oxford, UK, 1999.

[14] W. Shoutens, Stochastic Processes and Orthogonal Polynomials, Springer-Verlag, New York, 2000.

[15] A. TAflove, Computational Electrodynamics-The Finite-Difference Time-Domain Method, Artech House, Boston, 1995.

[16] N. Wiener, The homogeneous chaos, Amer. J. Math., 60 (1938), pp. 897-936.

[17] D. Xiu And J. S. Hesthaven, High-order collocation methods for differential equations with random inputs, SIAM J. Sci. Comput., 27 (2005), pp. 1118-1139.

[18] D. Xiu And G. E. Karniadakis, Modeling uncertainty in flow simulations via polynomial chaos, J. Comput. Phys., 187 (2003), pp. 137-167. 